\title{
A boundary element model for structural health monitoring using piezoelectric transducers
}

\section{F Zou, I Benedetti ${ }^{1}$ and M H Aliabadi}

Department of Aeronautics, Imperial College London, South Kensington Campus, London SW7 2AZ, UK

E-mail: f.zou11@imperial.ac.uk

Received 11 July 2013, in final form 15 November 2013

Published

\begin{abstract}
In this paper, for the first time, the boundary element method (BEM) is used for modelling smart structures instrumented with piezoelectric actuators and sensors. The host structure and its cracks are formulated with the 3D dual boundary element method (DBEM), and the modelling of the piezoelectric transducers implements a 3D semi-analytical finite element approach. The elastodynamic analysis of the structure is performed in the Laplace domain and the time history is obtained by inverse Laplace transform. The sensor signals obtained from BEM simulations show excellent agreement with those from FEM simulations and experiments. This work provides an alternative methodology for modelling smart structures in structural health monitoring (SHM) applications.
\end{abstract}

Keywords: boundary element method, smart structure, piezoelectric transducer, structural health monitoring, crack detection, wave propagation

(Some figures may appear in colour only in the online journal)
(Ed: Editor12)

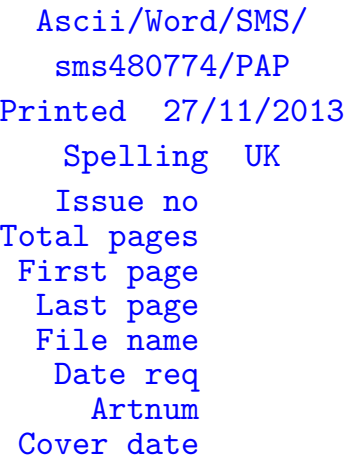

Ascii/Word/SMS/

rinted 27/11/2013

Spelling UK

Last page

Cover date

\section{Introduction}

In engineering applications, SHM helps to ensure the safety and the reliability of structures. Generally speaking, while structures in service are highly susceptible to both external and internal hazardous conditions (e.g. foreign object impacts, fatigue loadings, etc), SHM techniques are able to monitor the occurrences of these hazards and to detect the existences of any resultant damages done to the structures, before catastrophic failures happen.

Among the available transducers for SHM, lead zirconate titanate (PZT) piezoelectric transduction patches have attracted much attention for real-time in-service monitoring purposes, due to their light weight, high sensitivity, ability to actuate and sense ultrasonic guided waves (UGWs), and potential to form transducer networks. They have been used

1 On leave from: Dipartimento di Ingegneria Civile, Ambientale, Aerospaziale e dei Materiali, Università degli Studi di Palermo, Viale delle Scienze, Edificio 8, I-90128, Palermo, Italy. extensively for the detection of damages and impacts on various types of material and structure [1-7].

The development of a feasible SHM technique necessitates multi-disciplinary knowledge, ranging from structural mechanics to signal processing. In particular, a valid and reliable mathematical model of the structure under inspection would provide valuable assistance in understanding the response of the structure. A broad review of the models of smart structures with piezoelectric transducers can be found in [8]. Crawley and de Luis [9] studied analytically the static strain fields, induced by both surface-bonded and embedded piezoelectric actuators, in one-dimensional beams. Crawley and Lazarus [10] then extended this work into two-dimensional isotropic and anisotropic plates. Raghavan and Cesnik [11] introduced a dynamic model for the piezoelectric actuation and reception and the propagation of UGWs in plates. Lin and Yuan [12] proposed a static formulation for piezoelectric actuators and sensors and coupled it with a dynamic wave propagation model for Lamb wave based damage detection for isotropic plates. 
In this work, the dynamic response of a 3D structure with surface-mounted piezoelectric transducers and cracks is solved with BEM. Compared to FEM, BEM only requires discretization of the boundaries of structures. This leads to significant reduction in the complexity of meshing, especially for less regular structures. Also, since the unknown parameters - traction and displacement-are only computed on the boundaries, the systems of equations are much smaller, resulting in less demand on computational resources. Furthermore, with boundary discretization, the continuity of the interior of structures is preserved. Consequently, the solutions of internal points, which are obtained by interpolating boundary values, keep high resolution [13]. In recent years, with the development of DBEM, BEM has become a preferred method for modelling cracks in both two- [14] and three-dimensional [15, 16] domains. In DBEM, each crack is treated as an additional pair of boundaries, allowing for the calculation of stress intensity at crack tips and the prediction of crack growth paths. The book by Aliabadi [13] provides a comprehensive encyclopaedia of the types of problem that have been solved with BEM.

In terms of modelling and solving for piezoelectric applications with BEM, much research has been devoted to investigating the behaviour of piezoelectric material itself $[17,18]$. For modelling piezoelectric smart structures in SHM, Leme et al [19] established a static model for the analysis of $2 \mathrm{D}$ plates bonded with piezoelectric sensors which are formulated as beams. In a later model developed by Benedetti et al [20], both the host structures and the piezoelectric sensors are three dimensional. On the dynamic side, Alaimo et al [21] proposed a 2D model for detecting delaminations in composite panels using piezoelectric sensors. In all of the abovementioned works, the excitations of the structures, which are required for generating sensor signals, depend on the application of mechanical loads. The lack of actuators which can be used while the structures are in service alienates these techniques from the concept of real-time monitoring.

The formulation presented in this paper extends the work done by Benedetti et al [20] into the dynamic regime. For the first time, BEM is used for modelling the actuation, the propagation and the reception of UGWs, which can be used for the detection and the characterization of defects, using piezoelectric transducers. The solid structure and its cracks are formulated with 3D DBEM. The 3D models for piezoelectric actuators and sensors are developed by taking into account the full electro-mechanical coupling and the relevant boundary conditions. They are expressed in terms of the relationship between voltage and BEM variables, i.e. displacement and traction, to allow for straightforward coupling with the foundation. The elastodynamic analysis is carried out in Laplace domain, in which the boundary integral equations are solved for a number of Laplace parameters. The corresponding response in the time domain is acquired by inverse Laplace transform. Finally, the sensor signals obtained from BEM and FEM simulations and experiments, for both pristine and cracked structures, demonstrated exceptional coherence.

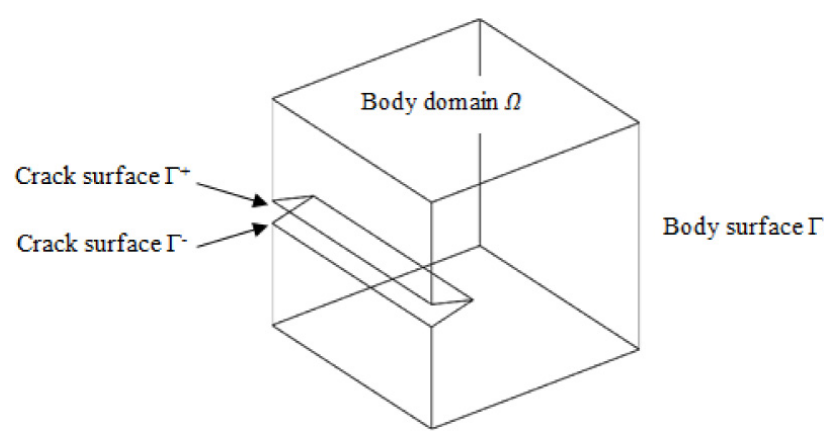

Figure 1. A 3D elastic body with a crack.

\section{Dual boundary element method}

Consider an elastic isotropic body with a crack as shown in figure 1 . The outer boundary of the body is given by $\Gamma$ and the crack is described by the boundaries $\Gamma^{+}$and $\Gamma^{-}$. Assuming that there are no body forces, the dynamics of the body in the time domain is governed by the Navier-Cauchy equation such that

$$
\begin{gathered}
c_{2}^{2} u_{i, k k}(\boldsymbol{x}, t)+\left(c_{1}^{2}-c_{2}^{2}\right) u_{k, k i}(\boldsymbol{x}, t)=\ddot{u}_{i}(\boldsymbol{x}, t) \\
\boldsymbol{x}=(x, y, z) \in \Omega \quad i, k=1,2,3
\end{gathered}
$$

where $c_{1}$ and $c_{2}$ are the velocities of the longitudinal and the shear waves, and $u_{i}$ is the displacement of the body. The Laplace transform of equation (1) is given by

$$
c_{2}^{2} u_{i, k k}(\boldsymbol{x}, s)+\left(c_{1}^{2}-c_{2}^{2}\right) u_{k, i k}(\boldsymbol{x}, s)=s^{2} u_{i}(\boldsymbol{x}, s) .
$$

The solution of equation (2) has to satisfy the following boundary conditions:

$$
\begin{aligned}
u_{i}(\boldsymbol{x}, s) & =p_{i}(\boldsymbol{x}, s) & & \boldsymbol{x} \in \Gamma \\
t_{i}(\boldsymbol{x}, s) & =q_{i}(\boldsymbol{x}, s) & & \boldsymbol{x} \in \Gamma
\end{aligned}
$$

where $t_{i}$ is the traction, and $p_{i}$ and $q_{i}$ are the known values of displacements and tractions.

With the assumption that displacements and strains are continuous over the boundary, the solutions of equation (2), using BEM formulation, are written as [22]

$$
\begin{aligned}
& c_{i j}\left(\boldsymbol{x}^{\prime}\right) u_{j}\left(\boldsymbol{x}^{\prime}\right)+\int_{\Gamma} T_{i j}\left(\boldsymbol{x}^{\prime}, \boldsymbol{x}, s\right) u_{j}(\boldsymbol{x}) \mathrm{d} \Gamma \\
& \quad=\int_{\Gamma} U_{i j}\left(\boldsymbol{x}^{\prime}, \boldsymbol{x}, s\right) t_{j}(\boldsymbol{x}) \mathrm{d} \Gamma \\
& \frac{1}{2} t_{j}\left(\boldsymbol{x}^{\prime}\right)+n_{j}\left(\boldsymbol{x}^{\prime}\right) \int_{\Gamma} T_{k i j}\left(\boldsymbol{x}^{\prime}, \boldsymbol{x}, s\right) u_{k}(\boldsymbol{x}) \mathrm{d} \Gamma \\
& \quad=n_{i}\left(\boldsymbol{x}^{\prime}\right) \int_{\Gamma} U_{k i j}\left(\boldsymbol{x}^{\prime}, \boldsymbol{x}, s\right) t_{k}(\boldsymbol{x}) \mathrm{d} \Gamma
\end{aligned}
$$

where equations (4) and (5) are referred to as the displacement and the traction boundary integral equations. Also, $U_{i j}\left(\boldsymbol{x}^{\prime}, \boldsymbol{x}, s\right), U_{k i j}\left(\boldsymbol{x}^{\prime}, \boldsymbol{x}, s\right), T_{i j}\left(\boldsymbol{x}^{\prime}, \boldsymbol{x}, s\right)$ and $T_{k i j}\left(\boldsymbol{x}^{\prime}, \boldsymbol{x}, s\right)$, whose details are given in appendix A, are known as the Laplace transformed fundamental solutions and kernels of displacement and traction in elastodynamics. Moreover, in 
these equations, $\boldsymbol{x}^{\prime}$ is the field point and is used as the collocation point, $\boldsymbol{x}$ is the source point, and $n_{i}$ is the outward normal. $c_{i j}$ varies with the location of the collocation point, and for collocation points on smooth surfaces $c_{i j}=\frac{1}{2} \delta_{i j}$, in which $\delta_{i j}$ is the Kronecker delta.

In terms of numerical implementation, equation (4) is collocated at the outer boundary and at one of the crack surfaces, and equation (5) is at the other crack surface. For collocations at the crack surfaces, equations (4) and (5) adopt the following forms:

$$
\begin{aligned}
& c_{i j}\left(\boldsymbol{x}_{0}^{-}\right) u_{j}\left(\boldsymbol{x}_{0}^{-}\right)+c_{i j}\left(\boldsymbol{x}_{0}^{+}\right) u_{j}\left(\boldsymbol{x}_{0}^{+}\right)+\int_{\Gamma} T_{i j}\left(\boldsymbol{x}_{0}^{-}, \boldsymbol{x}, s\right) \\
& \times u_{j}(\boldsymbol{x}) \mathrm{d} \Gamma(\boldsymbol{x})=\int_{\Gamma} U_{i j}\left(\boldsymbol{x}_{0}^{-}, \boldsymbol{x}, s\right) t_{j}(\boldsymbol{x}) \mathrm{d} \Gamma \\
& \frac{1}{2} t_{j}\left(\boldsymbol{x}_{0}^{+}\right)-\frac{1}{2} t_{j}\left(\boldsymbol{x}_{0}^{-}\right)+n_{j}\left(\boldsymbol{x}_{0}^{+}\right) \int_{\Gamma} T_{k i j}\left(\boldsymbol{x}_{0}^{+}, \boldsymbol{x}, s\right) u_{k}(\boldsymbol{x}) \mathrm{d} \Gamma \\
& =n_{i}\left(\boldsymbol{x}_{0}^{+}\right) \int_{\Gamma} U_{k i j}\left(\boldsymbol{x}_{0}^{+}, \boldsymbol{x}, s\right) t_{k}(\boldsymbol{x}) \mathrm{d} \Gamma
\end{aligned}
$$

where $\boldsymbol{x}_{0}^{-}$and $\boldsymbol{x}_{0}^{+}$are the points on the crack surface at which displacement and traction are collocated respectively. In order to perform the integrations in the boundary integral equations, the boundary of the body, including the crack surfaces, is discretized into eight-node quadrilateral elements.

After carrying out the collocations and the integrations, a linear system of equations results:

$$
\boldsymbol{H}(s) \tilde{\boldsymbol{u}}(s)=\boldsymbol{G}(s) \tilde{\boldsymbol{t}}(s)
$$

where the tildes indicate discretized nodal values. By applying boundary conditions, equation (8) can be rearranged into

$$
\boldsymbol{A}(s) \tilde{\boldsymbol{x}}(s)=\tilde{\boldsymbol{y}}(s)
$$

where $\boldsymbol{A}$ is the collocation matrix, and $\tilde{\boldsymbol{x}}$ contains the unknown nodal values and $\tilde{\boldsymbol{y}}$ the known nodal values.

\section{Piezoelectric transducer model}

In section 2, the formulation of DBEM for the host structure was introduced. It is now necessary to develop a piezoelectric transducer model which can be coupled with the host structure.

\subsection{Basic equations}

Consider a piezoelectric transducer, whose top and bottom surfaces are perpendicular to the electric poling direction, i.e. the $x_{3}$-direction. The elastic strain-displacement and the electric relationships, in tensor notation, are described by

$$
\begin{aligned}
& \gamma_{i j}=\frac{1}{2}\left(u_{i, j}+u_{j, i}\right) \quad i, j=1,2,3 \\
& E_{i}=-V_{, i} \quad i=1,2,3
\end{aligned}
$$

where $\gamma_{i j}$ is the strain, $E_{i}$ is the electric field and $V$ is the electric potential in the $x_{3}$-direction. Also, the electro-mechanical coupled relationships for piezoelectric materials are given by

$$
\begin{array}{ll}
\sigma_{i j}=C_{i j k l} \gamma_{k l}+e_{i j k} E_{k} & i, j, k, l=1,2,3 \\
D_{i}=e_{i k l} \gamma_{k l}+\varepsilon_{i k} E_{k} & i, k, l=1,2,3
\end{array}
$$

where $\sigma_{i j}$ is the stress, $D_{i}$ is the electric displacement, and $C_{i j k l}, e_{i j k}$ and $\varepsilon_{i k}$ are the elastic, the piezoelectric and the dielectric constants.

Equation (10) can be rewritten in matrix form as

$$
\left[\begin{array}{c}
\boldsymbol{\Gamma}_{p} \\
\boldsymbol{\Gamma}_{z}
\end{array}\right]=\left[\begin{array}{c}
\boldsymbol{D}_{\alpha} \\
\boldsymbol{D}_{\beta}+\boldsymbol{I} \frac{\partial}{\partial x_{3}}
\end{array}\right] \boldsymbol{U}
$$

where $\quad \boldsymbol{\Gamma}_{p}=\left[\begin{array}{lllll}\gamma_{11} & \gamma_{22} & \gamma_{12} & -E_{1} & -E_{2}\end{array}\right]^{\mathrm{T}}$ and $\boldsymbol{\Gamma}_{z}=$ $\left[\begin{array}{llll}\gamma_{13} & \gamma_{23} & \gamma_{33} & -E_{3}\end{array}\right]^{\mathrm{T}}$ are the generalized in-plane and out-ofplane strains, $\boldsymbol{U}=\left[\begin{array}{llll}u_{1} & u_{2} & u_{3} & V\end{array}\right]^{\mathrm{T}}$ is the generalized displacement, and $\boldsymbol{D}_{\alpha}$ and $\boldsymbol{D}_{\beta}$ are linear differential operators, whose details are given in appendix B. Similarly, equation (11) can be represented by

$$
\left[\begin{array}{c}
\boldsymbol{\Sigma}_{p} \\
\boldsymbol{\Sigma}_{z}
\end{array}\right]=\left[\begin{array}{ll}
\boldsymbol{R}_{p p} & \boldsymbol{R}_{p z} \\
\boldsymbol{R}_{z p} & \boldsymbol{R}_{z z}
\end{array}\right]\left[\begin{array}{c}
\boldsymbol{\Gamma}_{p} \\
\boldsymbol{\Gamma}_{z}
\end{array}\right]
$$

where $\quad \boldsymbol{\Sigma}_{p}=\left[\begin{array}{lllll}\sigma_{11} & \sigma_{22} & \sigma_{12} & D_{1} & D_{2}\end{array}\right]^{\mathrm{T}}$ and $\boldsymbol{\Sigma}_{z}=$ $\left[\begin{array}{llll}\sigma_{13} & \sigma_{23} & \sigma_{33} & D_{3}\end{array}\right]^{\mathrm{T}}$ are the generalized in-plane and outof-plane stresses, and $\boldsymbol{R}_{p p}, \boldsymbol{R}_{p z}, \boldsymbol{R}_{z p}$ and $\boldsymbol{R}_{z z}$, are the corresponding components of the elastic, the piezoelectric and the dielectric constants.

\subsection{Elemental state-space equation}

In order to derive the dynamic elemental state-space equation, a hybrid generalized functional for 3D piezoelectric materials is introduced [23]:

$\Pi=\int_{\Omega} \boldsymbol{\omega}\left(\boldsymbol{U}, \boldsymbol{\Sigma}_{z}\right) \mathrm{d} \Omega-\int_{\Gamma} \boldsymbol{T}^{\mathrm{T}}(\boldsymbol{U}-\overline{\boldsymbol{U}}) \mathrm{d} \Gamma-\int_{\Gamma} \boldsymbol{U}^{\mathrm{T}} \overline{\boldsymbol{T}} \mathrm{d} \Gamma$

where $\Omega$ and $\Gamma$ are the volume and the surface of the material, $\boldsymbol{\omega}\left(\boldsymbol{U}, \boldsymbol{\Sigma}_{z}\right)$ is the energy density, whose details are given in appendix B, $\boldsymbol{T}=\left[\begin{array}{llll}t_{1} & t_{2} & t_{3} & D_{n}\end{array}\right]^{\mathrm{T}}$ is the generalized traction, and the overlines indicate boundary values.

The piezoelectric material is discretized into eight-node quadrilateral elements, like the host structure, such that

$$
\left[\begin{array}{c}
\boldsymbol{U} \\
\boldsymbol{\Sigma}_{z}
\end{array}\right]=\left[\begin{array}{cc}
\boldsymbol{N}(\xi, \eta) & \mathbf{0} \\
\mathbf{0} & \boldsymbol{N}(\xi, \eta)
\end{array}\right]\left[\begin{array}{c}
\tilde{\boldsymbol{U}} \\
\tilde{\boldsymbol{\Sigma}}_{z}
\end{array}\right]=\tilde{\boldsymbol{N}}(\xi, \eta) \tilde{\boldsymbol{Y}}
$$

where $\quad \tilde{\boldsymbol{U}}\left(=\left[\begin{array}{ll}\tilde{\boldsymbol{u}}^{\mathrm{T}} & \tilde{\boldsymbol{V}}^{\mathrm{T}}\end{array}\right]^{\mathrm{T}}=\left[\begin{array}{llll}\tilde{\boldsymbol{u}}_{1}^{\mathrm{T}} & \tilde{\boldsymbol{u}}_{2}^{\mathrm{T}} & \tilde{\boldsymbol{u}}_{3}^{\mathrm{T}} & \tilde{\boldsymbol{V}}^{\mathrm{T}}\end{array}\right]^{\mathrm{T}}\right)$ and $\tilde{\boldsymbol{\Sigma}}(=$ $\left.\left[\begin{array}{ll}\tilde{\boldsymbol{\sigma}}^{\mathrm{T}} & \tilde{\boldsymbol{D}}^{\mathrm{T}}\end{array}\right]^{\mathrm{T}}=\left[\begin{array}{llll}\tilde{\boldsymbol{\sigma}}_{13}^{\mathrm{T}} & \tilde{\boldsymbol{\sigma}}_{23}^{\mathrm{T}} & \tilde{\boldsymbol{\sigma}}_{33}^{\mathrm{T}} & \tilde{\boldsymbol{D}}_{3}^{\mathrm{T}}\end{array}\right]^{\mathrm{T}}\right)$ contain the nodal values of generalized displacements and out-of-plane stresses. Moreover, in equation (15),

$$
\boldsymbol{N}(\xi, \eta)=\left[\begin{array}{cccc}
\boldsymbol{N}_{\mathrm{s}} & \mathbf{0} & \mathbf{0} & \mathbf{0} \\
\mathbf{0} & \boldsymbol{N}_{\mathrm{s}} & \mathbf{0} & \mathbf{0} \\
\mathbf{0} & \mathbf{0} & \boldsymbol{N}_{\mathrm{s}} & \mathbf{0} \\
\mathbf{0} & \mathbf{0} & \mathbf{0} & \boldsymbol{N}_{\mathrm{s}}
\end{array}\right]
$$


where $N_{\mathrm{s}}$ is a $(1 \times 8)$ matrix that contains the shape functions of eight-node quadrilateral elements.

From the first variation $(\delta \Pi=0)$ with respect to the variables $\boldsymbol{\Sigma}_{z}$ and $\tilde{\boldsymbol{U}}$, the following state-space equation is obtained:

$$
\boldsymbol{P} \frac{\mathrm{d} \tilde{Y}\left(x_{3}\right)}{\mathrm{d} x_{3}}=\boldsymbol{Q} \tilde{\boldsymbol{Y}}\left(x_{3}\right)
$$

where

$$
\begin{aligned}
& \boldsymbol{P}=\int_{s} \tilde{\boldsymbol{N}}^{\mathrm{T}}(\xi, \eta) \tilde{\boldsymbol{N}}(\xi, \eta) J(\xi, \eta) \mathrm{d} \xi \mathrm{d} \eta \\
& \boldsymbol{Q}=\int_{s}\left[\begin{array}{ll}
\boldsymbol{Q}_{11} & \boldsymbol{Q}_{12} \\
\boldsymbol{Q}_{21} & \boldsymbol{Q}_{22}
\end{array}\right] J(\xi, \eta) \mathrm{d} \xi \mathrm{d} \eta .
\end{aligned}
$$

The details of $\boldsymbol{Q}_{i j}$ are given in appendix B. $J(\xi, \eta)$ is the determinant of the Jacobian matrix.

Finally, by performing integration on equation (16), the resultant expression can be found:

$$
\tilde{\boldsymbol{Y}}\left(x_{3}\right)=\exp \left(\boldsymbol{P}^{-1} \boldsymbol{Q} x_{3}\right) \tilde{\boldsymbol{Y}}(0) .
$$

For a single-layered piezoelectric material, equation (17) can be expanded into

$$
\left[\begin{array}{c}
\tilde{\boldsymbol{u}}_{\mathrm{t}} \\
\tilde{\boldsymbol{V}}_{\mathrm{t}} \\
\tilde{\boldsymbol{\sigma}}_{\mathrm{t}} \\
\tilde{\boldsymbol{D}}_{\mathrm{t}}
\end{array}\right]=\left[\begin{array}{llll}
\boldsymbol{L}_{u u}(h) & \boldsymbol{L}_{u V}(h) & \boldsymbol{L}_{u \sigma}(h) & \boldsymbol{L}_{u D}(h) \\
\boldsymbol{L}_{V u}(h) & \boldsymbol{L}_{V V}(h) & \boldsymbol{L}_{V \sigma}(h) & \boldsymbol{L}_{V D}(h) \\
\boldsymbol{L}_{\sigma u}(h) & \boldsymbol{L}_{\sigma V}(h) & \boldsymbol{L}_{\sigma \sigma}(h) & \boldsymbol{L}_{\sigma D}(h) \\
\boldsymbol{L}_{D u}(h) & \boldsymbol{L}_{D V}(h) & \boldsymbol{L}_{D \sigma}(h) & \boldsymbol{L}_{D D}(h)
\end{array}\right]\left[\begin{array}{c}
\tilde{\boldsymbol{u}}_{\mathrm{b}} \\
\tilde{\boldsymbol{V}}_{\mathrm{b}} \\
\tilde{\boldsymbol{\sigma}}_{\mathrm{b}} \\
\tilde{\boldsymbol{D}}_{\mathrm{b}}
\end{array}\right]
$$

where the subscripts $t$ and $b$ indicate the top and the bottom surfaces of the piezoelectric transducer, and $h$ is the thickness of the material.

The models for piezoelectric actuators and sensors can now be acquired by enforcing the relevant boundary conditions.

\subsection{Actuator model}

Since electric potential is a relative quantity, the bottom surfaces of piezoelectric transducers are often made the reference surface with zero potential. This gives rise to an electric boundary condition such that

$$
\tilde{\boldsymbol{V}}_{\mathrm{b}}=\mathbf{0} \text {. }
$$

Also, a mechanical boundary condition, which comes from the fact that the top surfaces of piezoelectric transducers are stress free, can be expressed as

$$
\tilde{\boldsymbol{\sigma}}_{\mathrm{t}}=\mathbf{0} \text {. }
$$

By applying these boundary conditions in equation (18), the following relationships can be obtained:

$$
\begin{aligned}
& \tilde{\boldsymbol{V}}_{\mathrm{t}}=\boldsymbol{L}_{V u} \tilde{\boldsymbol{u}}_{\mathrm{b}}+\boldsymbol{L}_{V \sigma} \tilde{\boldsymbol{\sigma}}_{\mathrm{b}}+\boldsymbol{L}_{V D} \tilde{\boldsymbol{D}}_{\mathrm{b}} \\
& \tilde{\boldsymbol{\sigma}}_{\mathrm{t}}=\boldsymbol{L}_{\sigma u} \tilde{\boldsymbol{u}}_{\mathrm{b}}+\boldsymbol{L}_{\sigma \sigma} \tilde{\boldsymbol{\sigma}}_{\mathrm{b}}+\boldsymbol{L}_{\sigma D} \tilde{\boldsymbol{D}}_{\mathrm{b}}=\mathbf{0} .
\end{aligned}
$$

The manipulation of equations (21) and (22) yields

$$
\begin{aligned}
\tilde{\boldsymbol{\sigma}}_{\mathrm{b}}= & \left(\boldsymbol{L}_{\sigma \sigma}-\boldsymbol{L}_{\sigma D} \boldsymbol{L}_{V D}^{-1} \boldsymbol{L}_{V \sigma}\right)^{-1}\left(\boldsymbol{L}_{\sigma D} \boldsymbol{L}_{V D}^{-1} \boldsymbol{L}_{V u}-\boldsymbol{L}_{\sigma u}\right) \tilde{\boldsymbol{u}}_{\mathrm{b}} \\
& -\left(\boldsymbol{L}_{\sigma \sigma}-\boldsymbol{L}_{\sigma D} \boldsymbol{L}_{V D}^{-1} \boldsymbol{L}_{V \sigma}\right)^{-1} \boldsymbol{L}_{\sigma D} \boldsymbol{L}_{V D}^{-1} \tilde{\boldsymbol{V}}_{\mathrm{t}}
\end{aligned}
$$

In order to couple the actuator model with the host structure, which is formulated by BEM, stress needs to be converted into traction, and displacement, which is in the local coordinate, needs to be transformed into the global coordinate of the host structure. Therefore, by performing coordinate transformations, the following expression is found:

$$
\tilde{\boldsymbol{t}}_{\mathrm{b}}=\boldsymbol{\Psi}^{\mathrm{a}} \tilde{\boldsymbol{u}}_{\mathrm{b}}+\boldsymbol{\Phi} \tilde{\boldsymbol{V}}_{\mathrm{t}}
$$

where

$$
\begin{aligned}
& \boldsymbol{\Psi}^{\mathrm{a}}=-\boldsymbol{\Lambda}^{-\mathbf{1}}\left(\boldsymbol{L}_{\sigma \sigma}-\boldsymbol{L}_{\sigma D} \boldsymbol{L}_{V D}^{-1} \boldsymbol{L}_{V \sigma}\right)^{-1}\left(\boldsymbol{L}_{\sigma D} \boldsymbol{L}_{V D}^{-1} \boldsymbol{L}_{V u}-\boldsymbol{L}_{\sigma u}\right) \boldsymbol{\Lambda} \\
& \boldsymbol{\Phi}=\boldsymbol{\Lambda}^{-\mathbf{1}}\left(\boldsymbol{L}_{\sigma \sigma}-\boldsymbol{L}_{\sigma D} \boldsymbol{L}_{V D}^{-1} \boldsymbol{L}_{V \sigma}\right)^{-1} \boldsymbol{L}_{\sigma D} \boldsymbol{L}_{V D}^{-1} .
\end{aligned}
$$

In the above representations, $\Lambda$ is the rotation matrix.

\subsection{Sensor model}

In addition to the boundary conditions formulated for the actuator model, a couple of other boundary conditions are necessary for developing the sensor model. The top and the bottom surfaces of piezoelectric transducers are usually coated with thin metallic layers to ensure that equipotentiality is achieved on each surface. For the top surfaces, this is described by

$$
V_{\mathrm{t}_{1}}-V_{\mathrm{t}_{i}}=\boldsymbol{B} \tilde{\boldsymbol{V}}_{\mathrm{t}}=\mathbf{0} \quad i=2, \ldots, 7
$$

where $V_{\mathrm{t}_{i}}$ is the $i$ th node of the top surface and $\boldsymbol{B}$ is a $(7 \times 8)$ matrix.

Also, since piezoelectric sensors are not subject to external electric potential, the law of charge conservation in open circuits applies. On a discretized piezoelectric element, the following boundary condition is obtained:

$$
Q_{\text {top }}=\int_{A_{\text {top }}} \boldsymbol{N}_{\mathrm{s}}(\xi, \eta) J(\xi, \eta) \tilde{\boldsymbol{D}}_{\mathrm{t}} \mathrm{d} \xi \mathrm{d} \eta=\boldsymbol{b}^{\mathrm{T}} \tilde{\boldsymbol{D}}_{\mathrm{t}}=0
$$

where $A_{\text {top }}$ is the area of the top surface.

Using the boundary condition described by equation (19), another equation, in addition to equations (21) and (22), can be extracted from equation (18):

$$
\tilde{\boldsymbol{D}}_{\mathrm{t}}=\boldsymbol{L}_{D u} \tilde{\boldsymbol{u}}_{\mathrm{b}}+\boldsymbol{L}_{D \sigma} \tilde{\boldsymbol{\sigma}}_{\mathrm{b}}+\boldsymbol{L}_{D D} \tilde{\boldsymbol{D}}_{\mathrm{b}} .
$$

By enforcing the boundary conditions defined by equations (25) and (26), equations (21) and (27) can be rewritten as

$$
\begin{array}{r}
\boldsymbol{B}\left(\boldsymbol{L}_{V u} \tilde{\boldsymbol{u}}_{\mathrm{b}}+\boldsymbol{L}_{V \sigma} \tilde{\boldsymbol{\sigma}}_{\mathrm{b}}+\boldsymbol{L}_{V D} \tilde{\boldsymbol{D}}_{\mathrm{b}}\right)=\mathbf{0} \\
\boldsymbol{b}^{\mathrm{T}}\left(\boldsymbol{L}_{D u} \tilde{\boldsymbol{u}}_{\mathrm{b}}+\boldsymbol{L}_{D \sigma} \tilde{\boldsymbol{\sigma}}_{\mathrm{b}}+\boldsymbol{L}_{D D} \tilde{\boldsymbol{D}}_{\mathrm{b}}\right)=0 .
\end{array}
$$

Equations (28) and (29) can be combined into a single expression such that

$$
\left[\begin{array}{c}
\boldsymbol{B} \boldsymbol{L}_{V u} \\
\boldsymbol{b}^{\mathrm{T}} \boldsymbol{L}_{D u}
\end{array}\right] \tilde{\boldsymbol{u}}_{\mathrm{b}}+\left[\begin{array}{c}
\boldsymbol{B} \boldsymbol{L}_{V \sigma} \\
\boldsymbol{b}^{\mathrm{T}} \boldsymbol{L}_{D \sigma}
\end{array}\right] \tilde{\boldsymbol{\sigma}}_{\mathrm{b}}+\left[\begin{array}{c}
\boldsymbol{B} \boldsymbol{L}_{V D} \\
\boldsymbol{b}^{\mathrm{T}} \boldsymbol{L}_{D D}
\end{array}\right] \tilde{\boldsymbol{D}}_{\mathrm{b}}=\mathbf{0} .
$$

The manipulation of equations (21), (22) and (30) gives

$$
\begin{aligned}
\tilde{\boldsymbol{\sigma}}_{\mathrm{b}} & =-\hat{\boldsymbol{L}}_{\sigma \sigma}^{-1} \hat{\boldsymbol{L}}_{\sigma u} \tilde{\boldsymbol{u}}_{\mathrm{b}} \\
\tilde{\boldsymbol{V}}_{\mathrm{t}} & =\left(\hat{\boldsymbol{L}}_{V u}-\hat{\boldsymbol{L}}_{V \sigma} \hat{\boldsymbol{L}}_{\sigma \sigma}^{-1} \hat{\boldsymbol{L}}_{\sigma u}\right) \tilde{\boldsymbol{u}}_{\mathrm{b}} .
\end{aligned}
$$




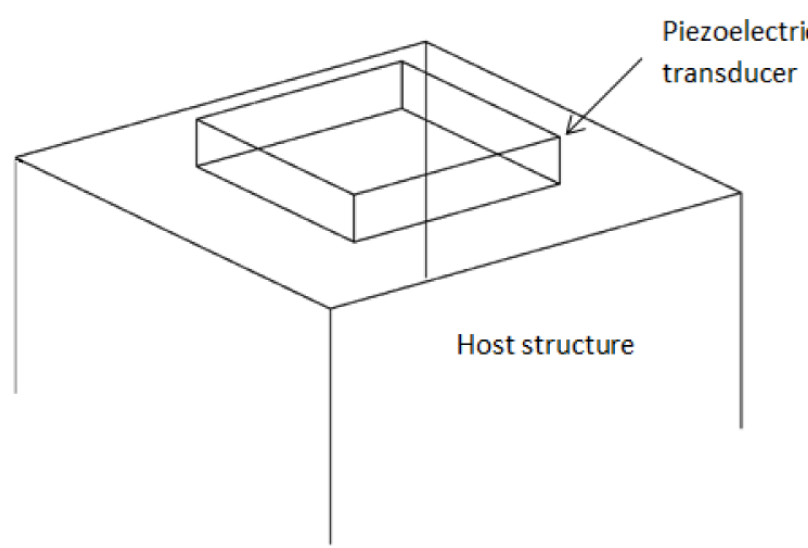

Figure 2. Coupling of piezoelectric transducer and host structure.

For coupling purpose, equations (31) and (32), after coordinate transformations, become

$$
\begin{aligned}
\tilde{\boldsymbol{t}}_{\mathrm{b}} & =\boldsymbol{\Psi}^{\mathrm{s}} \tilde{\boldsymbol{u}}_{\mathrm{b}} \\
\tilde{\boldsymbol{V}}_{\mathrm{t}} & =\boldsymbol{\Theta} \tilde{\boldsymbol{u}}_{\mathrm{b}}
\end{aligned}
$$

where

$$
\begin{aligned}
\boldsymbol{\Psi}^{\mathrm{s}} & =\boldsymbol{\Lambda}^{-\mathbf{1}} \hat{\boldsymbol{L}}_{\sigma \sigma}^{-1} \hat{\boldsymbol{L}}_{\sigma u} \boldsymbol{\Lambda} \\
\boldsymbol{\Theta} & =\left(\hat{\boldsymbol{L}}_{V u}-\hat{\boldsymbol{L}}_{V \sigma} \hat{\boldsymbol{L}}_{\sigma \sigma}^{-1} \hat{\boldsymbol{L}}_{\sigma u}\right) \boldsymbol{\Lambda} .
\end{aligned}
$$

The models for piezoelectric actuators and sensors, which are already in the Laplace domain due to the use of the functional described by equation (14), can now be coupled with the host structure via BEM variables-displacement and traction.

\section{Coupling of host structure and piezoelectric transducers}

Consider a piezoelectric patch, which is assumed to be perfectly bonded to the host structure, as shown in figure 2 . The presence of piezoelectric transducers on the boundary of the host structure modifies equation (8) such that

$$
\begin{gathered}
\boldsymbol{H}_{\mathrm{h}} \tilde{\boldsymbol{u}}_{\mathrm{h}}+\sum_{K_{\mathrm{a}}} \boldsymbol{H}_{i}^{k_{\mathrm{a}}} \tilde{\boldsymbol{u}}_{i}^{k_{\mathrm{a}}}+\sum_{K_{\mathrm{s}}} \boldsymbol{H}_{i}^{k_{\mathrm{s}}} \tilde{\boldsymbol{u}}_{i}^{k_{\mathrm{s}}} \\
=\boldsymbol{G}_{\mathrm{h}} \tilde{\boldsymbol{t}}_{\mathrm{h}}+\sum_{K_{\mathrm{a}}} \boldsymbol{G}_{i}^{k_{\mathrm{a}}} \tilde{\boldsymbol{t}}_{i}^{k_{\mathrm{a}}}+\sum_{K_{\mathrm{s}}} \boldsymbol{G}_{i}^{k_{\mathrm{s}}} \tilde{\boldsymbol{t}}_{i}^{k_{\mathrm{s}}}
\end{gathered}
$$

where $K_{\mathrm{a}}$ and $K_{\mathrm{s}}$ are the numbers of actuators and sensors respectively, the superscripts $k_{\mathrm{a}}$ and $k_{\mathrm{s}}$ indicate the $k$ th actuator and the $k$ th sensor, the subscript $i$ stands for the host structure at the interfaces with transducers, and the subscript $h$ refers to the rest of the host structure.

Due to the continuity of displacements and tractions at the interfaces, the following relationships can be deduced:

$$
\begin{aligned}
\tilde{\boldsymbol{u}}_{i}^{k} & =\tilde{\boldsymbol{u}}_{\mathrm{b}}^{k} \\
\tilde{\boldsymbol{t}}_{i}^{k} & =-\tilde{\boldsymbol{t}}_{\mathrm{b}}^{k}
\end{aligned}
$$

where the superscript $k$ indicate the $k$ th transducer, be it an actuator or a sensor.
By manipulating equations (24), (33), (36) and (37),

$$
\begin{aligned}
\tilde{\boldsymbol{t}}_{i}^{k_{\mathrm{a}}} & =-\left(\boldsymbol{\Psi}^{k_{\mathrm{a}}} \tilde{\boldsymbol{u}}_{i}^{k_{\mathrm{a}}}+\boldsymbol{\Phi} \tilde{\boldsymbol{V}}_{\mathrm{t}}\right) \\
\tilde{t}_{i}^{k_{\mathrm{s}}} & =-\boldsymbol{\Psi}^{k_{\mathrm{s}}} \tilde{\boldsymbol{u}}_{i}^{k_{\mathrm{s}}} .
\end{aligned}
$$

By substituting equations (38) and (39) into (35),

$$
\begin{aligned}
& \boldsymbol{H}_{\mathrm{h}} \tilde{\boldsymbol{u}}_{\mathrm{h}}+\sum_{K_{\mathrm{a}}}\left(\boldsymbol{H}_{i}^{k_{\mathrm{a}}}+\boldsymbol{G}_{i}^{k_{\mathrm{a}}} \boldsymbol{\Psi}^{k_{\mathrm{a}}}\right) \tilde{\boldsymbol{u}}_{i}^{k_{\mathrm{a}}}+\sum_{K_{\mathrm{s}}}\left(\boldsymbol{H}_{i}^{k_{\mathrm{s}}}+\boldsymbol{G}_{i}^{k_{\mathrm{s}}} \boldsymbol{\Psi}^{k_{\mathrm{s}}}\right) \tilde{\boldsymbol{u}}_{i}^{k_{\mathrm{s}}} \\
& =\boldsymbol{G}_{\mathrm{h}} \tilde{\boldsymbol{t}}_{\mathrm{h}}-\sum_{K_{\mathrm{a}}} \boldsymbol{G}_{i}^{k_{\mathrm{a}}} \boldsymbol{\Phi} \tilde{\boldsymbol{V}}_{\mathrm{t}}
\end{aligned}
$$

Equation (40) can be rearranged into

$$
\begin{gathered}
\boldsymbol{H}_{\mathrm{h}} \tilde{\boldsymbol{u}}_{\mathrm{h}}+\sum_{k_{\mathrm{a}}} \boldsymbol{H}_{i}^{k_{\mathrm{a}}} \tilde{\boldsymbol{u}}_{i}^{k_{\mathrm{a}}}+\sum_{k_{\mathrm{s}}} \boldsymbol{H}_{i}^{k_{\mathrm{s}}} \tilde{\boldsymbol{u}}_{i}^{k_{\mathrm{s}}}+\sum_{k_{\mathrm{a}}} \boldsymbol{G}_{i}^{k_{\mathrm{a}}} \boldsymbol{\Psi}^{k_{\mathrm{a}}} \tilde{\boldsymbol{u}}_{i}^{k_{\mathrm{a}}} \\
+\sum_{k_{\mathrm{s}}} \boldsymbol{G}_{i}^{k_{\mathrm{s}}} \boldsymbol{\Psi}^{k_{\mathrm{s}}} \tilde{\boldsymbol{u}}_{i}^{k_{\mathrm{s}}}=\boldsymbol{G}_{\mathrm{h}} \tilde{\boldsymbol{t}}_{\mathrm{h}}-\sum_{k_{\mathrm{a}}} \boldsymbol{G}_{i}^{k_{\mathrm{a}}} \boldsymbol{\Phi} \tilde{\boldsymbol{V}}_{\mathrm{t}}
\end{gathered}
$$

In equation (41), it can be seen that the first three terms on the left-hand side and the first term on the right-hand side assemble equation (9), and the other terms represent the contribution from the presence of piezoelectric transducers. Therefore, equation (41) can be rewritten in the form of equation (9) such that

$$
\begin{aligned}
& \boldsymbol{A} \tilde{\boldsymbol{x}}+\sum_{k_{\mathrm{a}}} \boldsymbol{G}_{i}^{k_{\mathrm{a}}} \boldsymbol{\Psi}^{k_{\mathrm{a}}} \tilde{\boldsymbol{u}}_{i}^{k_{\mathrm{a}}}+\sum_{k_{\mathrm{s}}} \boldsymbol{G}_{i}^{k_{\mathrm{s}}} \boldsymbol{\Psi}^{k_{\mathrm{s}}} \tilde{\boldsymbol{u}}_{i}^{k_{\mathrm{s}}} \\
& =\tilde{\boldsymbol{y}}-\sum_{k_{\mathrm{a}}} \boldsymbol{G}_{i}^{k_{\mathrm{a}}} \boldsymbol{\Phi} \tilde{\boldsymbol{V}}_{\mathrm{t} .}
\end{aligned}
$$

Equation (42) is then solved for a certain number of Laplace parameters by using an adaptive cross approximation (ACA) algorithm. Details of the ACA algorithm and its applications can be found in [24, 25]. The response of the structure in the time domain is obtained by the inverse Laplace transform, whose details are given in appendix C.

\section{Numerical results and experimental validation}

In this section, the sensor signals obtained from BEM simulations are compared with those from FEM simulations and experiments. Some parametric studies are then carried out with the experimentally validated model.

\subsection{Actuation signal}

In SHM applications, five-cycle Hanning-windowed sinusoidal tonebursts are often used as the diagnostic signals [12]. The formula of such a toneburst is written as

$f(t)=\frac{1}{2} V \sin \left(2 \pi f_{\mathrm{c}} t\right)\left[1-\cos \left(\frac{2 \pi f_{\mathrm{c}} t}{5}\right)\right] H\left(\frac{5}{f_{\mathrm{c}}}-t\right)$

where $V$ is the peak voltage, $f_{\mathrm{c}}$ is the central frequency and $H$ stands for Heaviside step function.

\subsection{Experimental setup}

The complete experimental setup, as shown in figure 4, consists of a controller, a signal generator and a data logger. 

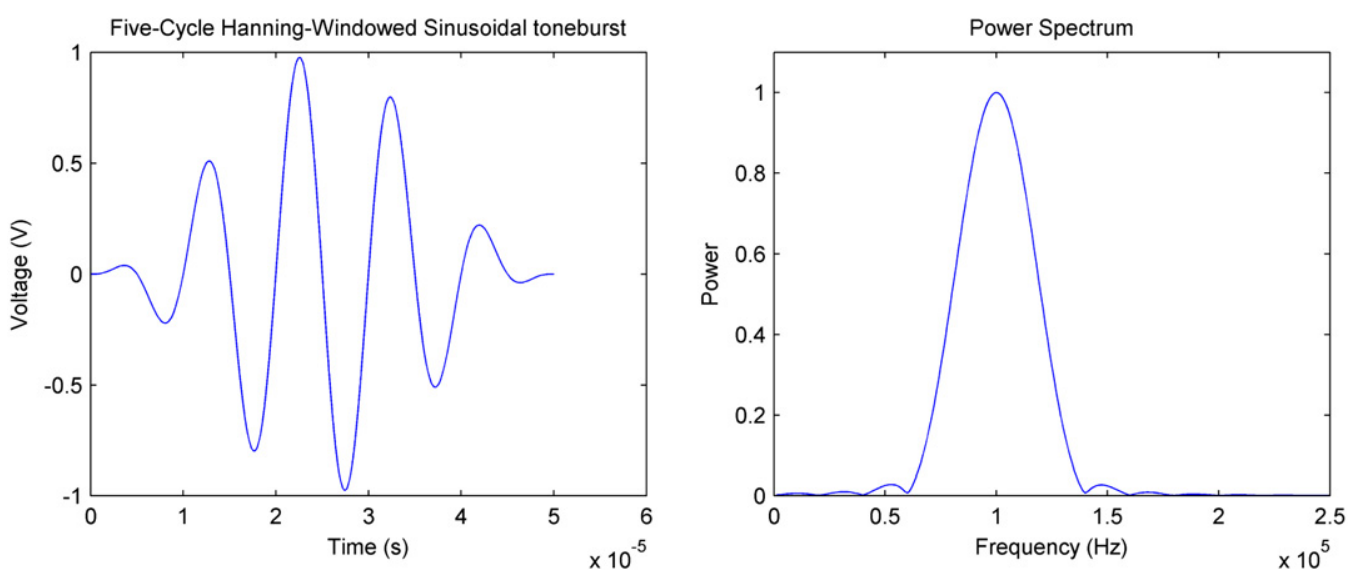

Figure 3. Time history and power spectrum of a five-cycle Hanning-windowed sinusoidal toneburst with $10 \mathrm{~V}$ peak voltage and $100 \mathrm{kHz}$ central frequency.

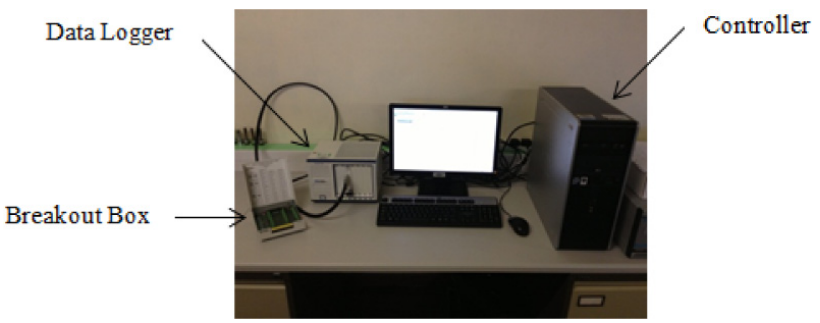

Figure 4. Experimental setup.

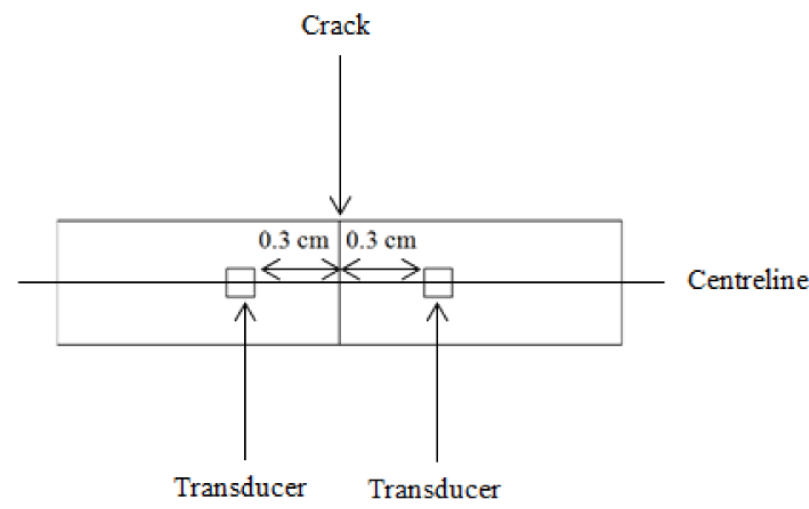

Figure 5. Arrangement of transducers and crack.

The controller, in this case, is a desktop computer with an Intel ${ }^{\circledR}$ Core $^{\mathrm{TM}} 2$ Duo CPU. The signal generation and the data acquisition are made possible by a National Instruments PXIe-6366 data acquisition card, which is capable of outputting signals at $3.33 \mathrm{MHz}$ and sampling them at $2 \mathrm{MHz}$. From experience, this data acquisition device is able to handle signals with frequencies up to $100 \mathrm{kHz}$.

\subsection{Specimens and details of numerical modelling}

Experiments have been conducted on a pristine and a cracked beam for validating the 3D DBEM. Both beams are made from aluminum and have dimensions of $200 \mathrm{~mm} \times 44 \mathrm{~mm} \times 37.5 \mathrm{~mm}$. The piezoelectric transducers,
Table 1. Material properties of aluminum beams.

\begin{tabular}{ll} 
Density $\left(\mathrm{kg} \mathrm{m}^{-3}\right)$ & 2700 \\
Young's modulus (GPa) & 70 \\
Poisson's ratio & 0.33 \\
\hline
\end{tabular}

which measure $10 \mathrm{~mm} \times 10 \mathrm{~mm} \times 1 \mathrm{~mm}$, are made from Noliac Group NCE51 piezoelectric ceramic and are attached to the beams by Loctite ${ }^{\circledR} 401$ superglue. The crack on one of the beams was manufactured by a wire-cut electric discharge machine in order to obtain a negligible distance of separation between the crack surfaces. The $0.1 \mathrm{~mm}$ wide crack runs across the width of the beam and is $18.75 \mathrm{~mm}$ deep.

The specimens used in experiments and their corresponding BEM models are shown in figure 6. The piezoelectric transducers are meshed with finer elements than the rest of the beam, because their model is essentially FEM based, and thus requires finer elements for convergence. The transition elements used around the piezoelectric transducers help to avoid numerical instabilities that could arise from sudden change of elements size.

Figure 7 compares the BEM and the FEM meshes of the host structure. As explained previously, for BEM discretization only happens on the boundaries of structures, and for 3D structures the boundaries refer to the external surfaces and internal crack surfaces. On the other hand, FEM requires discretization of the whole volumes of structures.

From figure 3 , it can be see that the frequency components of the actuation signal are spread over the range of $2 f_{\mathrm{c}}$. For inverse Laplace transform, the frequency increment is expressed as

$$
\Delta f=\frac{1}{T}
$$

Since it is known that the sensor signals will also contain the same frequency components [12], the number of Laplace parameters required is given by

$$
L=\frac{2 f_{\mathrm{c}}}{\Delta f}=2 f_{\mathrm{c}} T \text {. }
$$



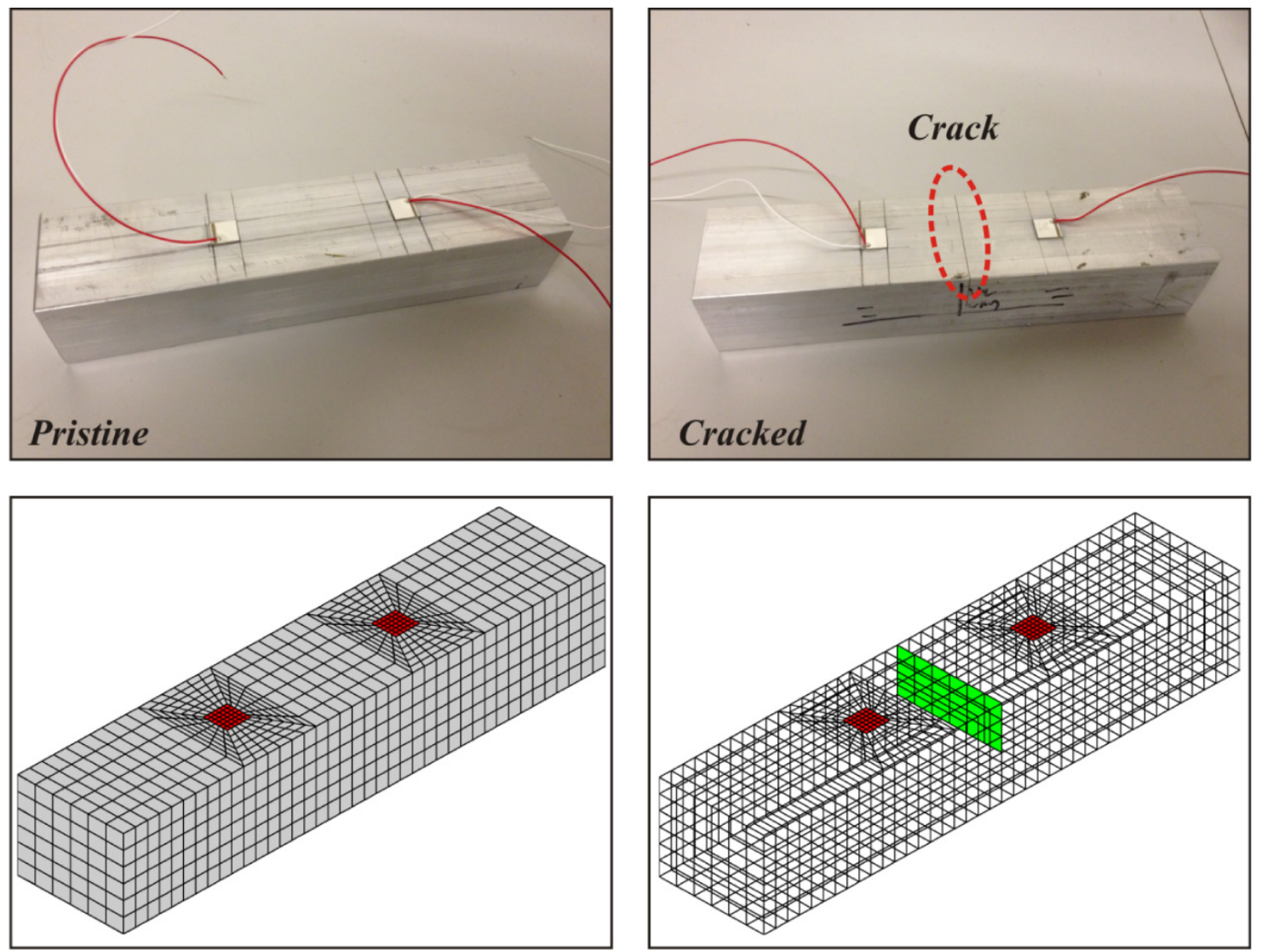

Figure 6. Experimental specimen and BEM model of pristine and cracked beams.

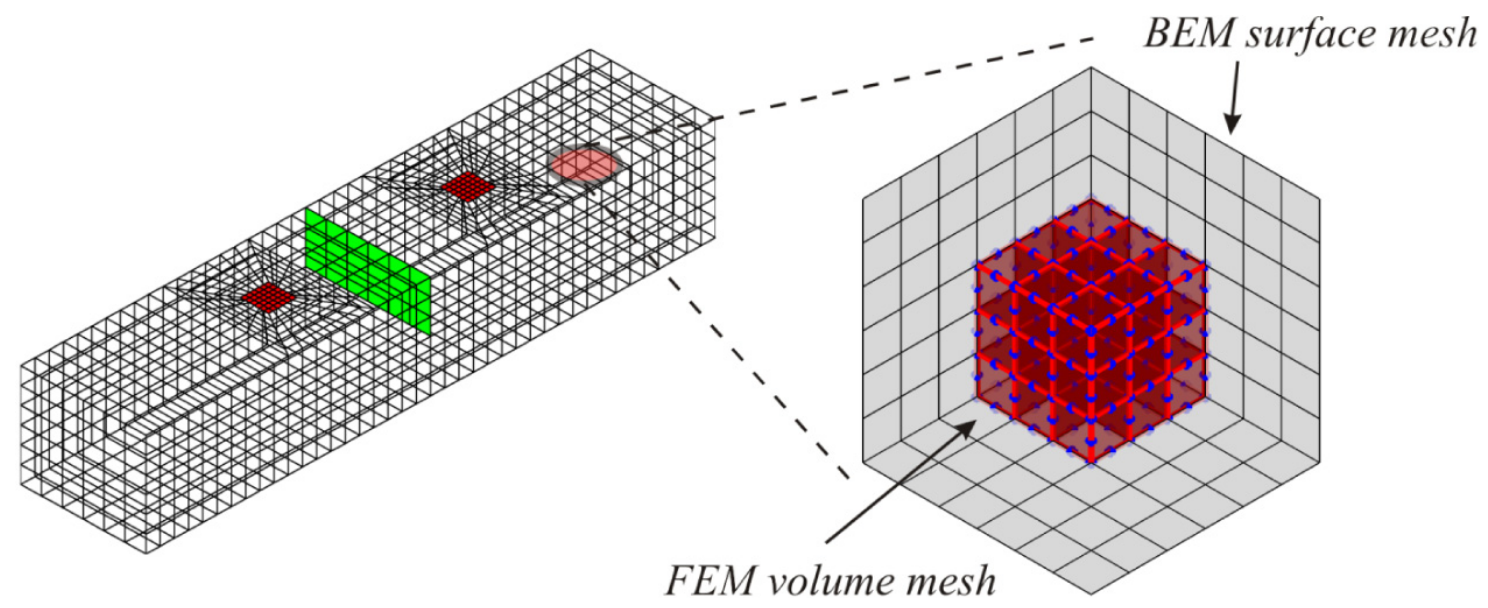

Figure 7. Comparison of BEM and FEM meshes.

Table 2. Material properties of piezoelectric transducers.

\begin{tabular}{|c|c|c|c|c|c|c|c|c|c|}
\hline Density $\left(\mathrm{kg} \mathrm{m}^{-3}\right)$ & 7800 & & & & & & & & \\
\hline Elastic (GPa) & $\begin{array}{l}C_{11} \\
129.22\end{array}$ & $\begin{array}{l}C_{22} \\
129.22\end{array}$ & $\begin{array}{l}C_{33} \\
116.90\end{array}$ & $\begin{array}{l}C_{12} \\
86.402\end{array}$ & $\begin{array}{l}C_{13} \\
83.062\end{array}$ & $\begin{array}{l}C_{23} \\
83.062\end{array}$ & $\begin{array}{l}C_{44} \\
28.830\end{array}$ & $\begin{array}{l}C_{55} \\
28.830\end{array}$ & $\begin{array}{l}C_{66} \\
21.410\end{array}$ \\
\hline Dielectric $\left(\mathrm{nF} \mathrm{m}^{-1}\right)$ & $\begin{array}{l}D_{11} \\
11.068\end{array}$ & $\begin{array}{l}D_{22} \\
11.068\end{array}$ & $\begin{array}{l}D_{33} \\
6.6406\end{array}$ & & & & & & \\
\hline Piezoelectric $\left(\mathrm{C} \mathrm{m}^{-2}\right)$ & $\begin{array}{c}e_{31} \\
-3.3831\end{array}$ & $\begin{array}{c}e_{32} \\
-3.3831\end{array}$ & $\begin{array}{l}e_{33} \\
16.520\end{array}$ & $\begin{array}{l}e_{24} \\
15.149\end{array}$ & $\begin{array}{l}e_{15} \\
15.149\end{array}$ & & & & \\
\hline
\end{tabular}




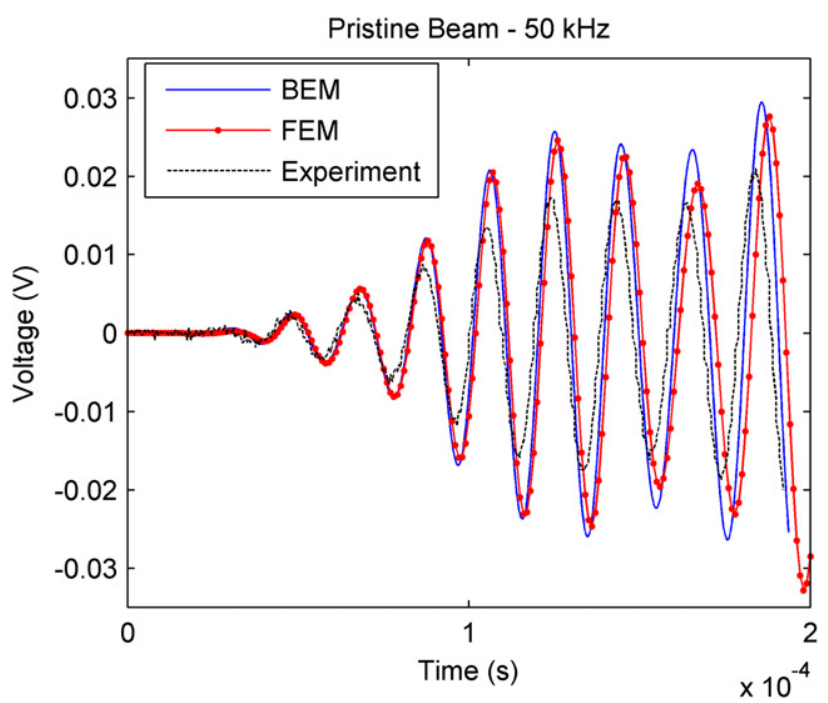

Figure 8. Validation of signals for pristine beam with $50 \mathrm{kHz}$ actuation frequency.

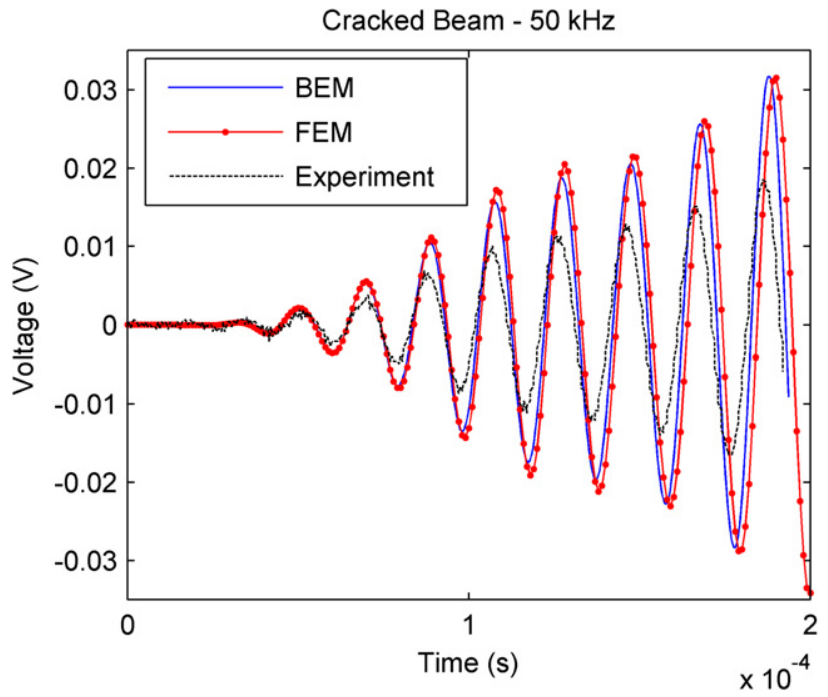

Figure 9. Validation of signals for cracked beam with $50 \mathrm{kHz}$ actuation frequency.

Benedetti et al [24] reported that the minimum wavelength of the fundamental solutions is found as

$$
\lambda=\sqrt{\frac{E}{2 \rho(1+v)}} \frac{T}{L}
$$

where $E, v$ and $\rho$ are the Young's modulus, the Poisson's ratio and the density of the material.

By substituting equation (45) into (46),

$$
\lambda=\sqrt{\frac{E}{2 \rho(1+v)}} \frac{1}{2 f_{\mathrm{c}}} .
$$

For discretization using eight-node quadrilateral elements, the element size of the host structure needs to be less than or equal to the value obtained by equation (47), so that the behaviours of the fundamental solutions can be captured.

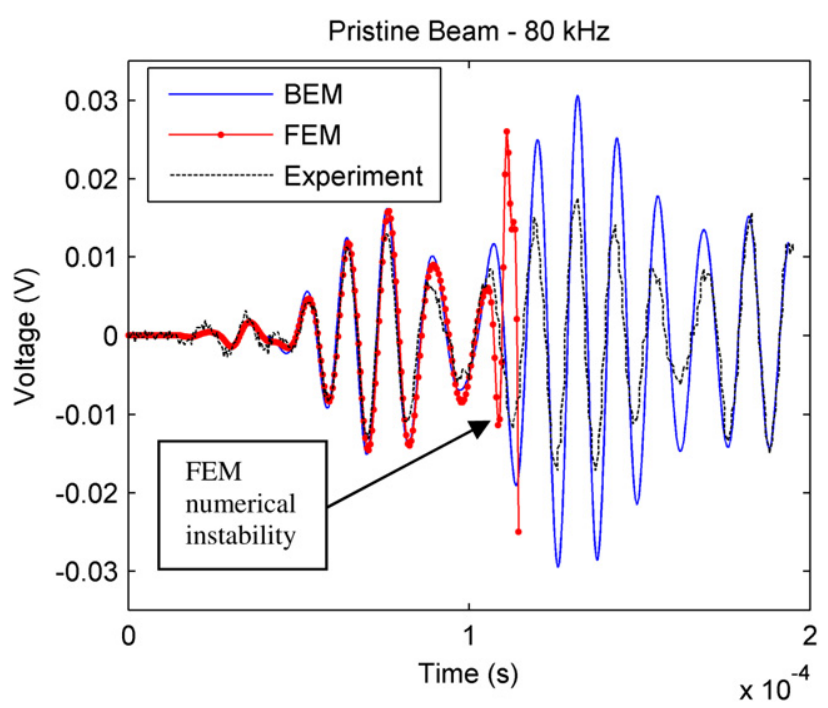

Figure 10. Validation of signals for pristine beam with $80 \mathrm{kHz}$ actuation frequency.

Table 3. Parameters for BEM simulations

\begin{tabular}{lll}
\hline & $50 \mathrm{kHz}$ & $80 \mathrm{kHz}$ \\
\hline$T(\mathrm{~s})$ & 0.0002 & 0.0002 \\
$L$ & 20 & 32 \\
Required element & 31.2 & 19.5 \\
size (mm) & & \\
\hline & & \\
Actual element size (mm) & $0.733 \times 5$ \\
& $0.625 \times 5$ \\
\hline
\end{tabular}

It can be seen from equations (45) and (47) that, as the central frequency of the actuation signal increases, the demands on computational time and memory are intensified since more Laplace parameters and smaller elements are required.

Actuation signals with two different central frequencies - 50 and $80 \mathrm{kHz}$ - are used for validating the model. Their corresponding simulation parameters are listed in table 3 .

Note that the choice of element size for the beams is constrained by the mesh of the piezoelectric transducers. For example, the width of the top surface needs to be divided into at least six elements in order to connect with the elements which construct the piezoelectric transducers.

\subsection{Sensor signals}

The sensor signals obtained by using BEM are compared with those from FEM and experiments. The FEM simulations are carried out by Abaqus ${ }^{\circledR} /$ Standard with implicit integration, which is known to be computationally expensive. However, since piezoelectric elements are not available in Abaqus ${ }^{\circledR} /$ Explicit, implicit integration is the only choice.

First of all, in all four figures, the signals obtained from BEM, FEM and experiments show excellent agreements. The fact that the results from BEM and FEM simulations match 


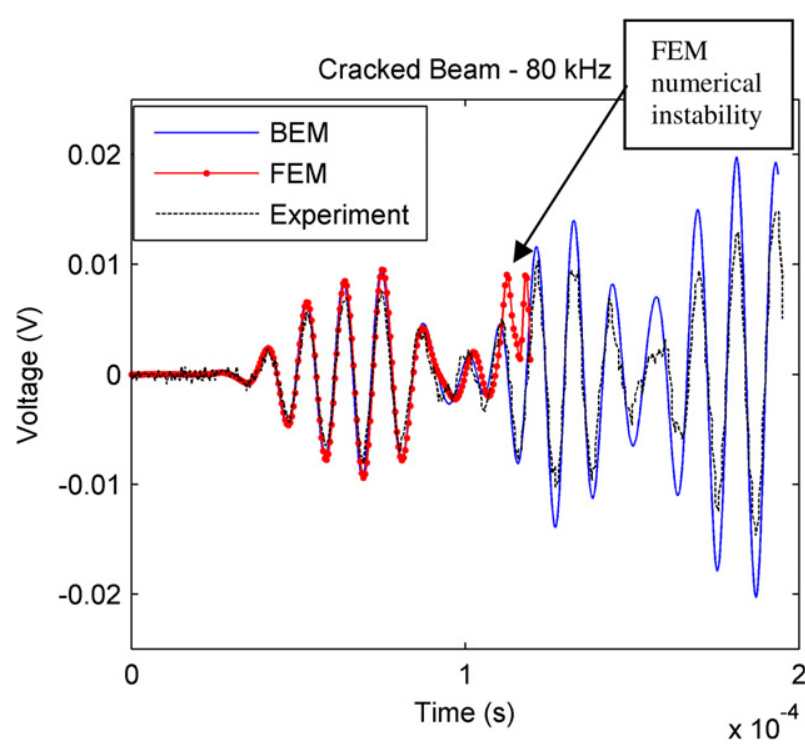

Figure 11. Validation of signals for cracked beam with $80 \mathrm{kHz}$ actuation frequency.

well in amplitude approves BEM as a numerical model. For signals with a central frequency of $50 \mathrm{kHz}$, slight delays in phase can be seen in the FEM results. These delays can be corrected by reducing the time increment and the element size, but the computational effort would become unaffordable. Also, if the reduction in time increment is not accompanied by an appropriate reduction in element size, as has been done in the cases with actuation signals of $80 \mathrm{kHz}$, the FEM simulations would begin to demonstrate divergence after a certain period of time. Furthermore, it is noticed that, for a different piezoelectric material, the divergence would happen later, indicating that FEM with implicit integration is potentially unstable for this type of application. Finally, for the simulation involving a pristine beam with an actuation signal of $80 \mathrm{kHz}$, the CPU times for FEM using Abaqus ${ }^{\circledR}$ and BEM are, respectively, 123743 and $10850 \mathrm{~s}$ on an Intel ${ }^{\circledR}$ Core $^{\mathrm{TM}} \mathrm{i7-2860QM}$ processor. It is worth mentioning that the BEM and its meshing algorithm are in-house codes and as such not optimized.

In comparison with the experimental results, some parts of the signals obtained from BEM simulations display higher amplitude and slight advance in phase. However, these phenomena are completely understandable because some of the conditions established in the model cannot be seamlessly satisfied in reality. For example, in the model, the presence of the adhesive layers, which contributes to phase delay due to shear lag, and material damping, which adds attenuation, are not considered. Also, the edges and the boundaries of the beams, if not machined to absolute precision, would affect the behaviour of the reflections. Furthermore, measurement errors, which may occur when cutting out the beams from bulk material and when attaching the piezoelectric transducers onto the beams, would lead to inconsistency of dimensions and thus phase shift in signals. In addition, the material properties of the aluminum and the piezoelectric ceramic may contain up to $10 \%$ deviation from the actual values, as advised

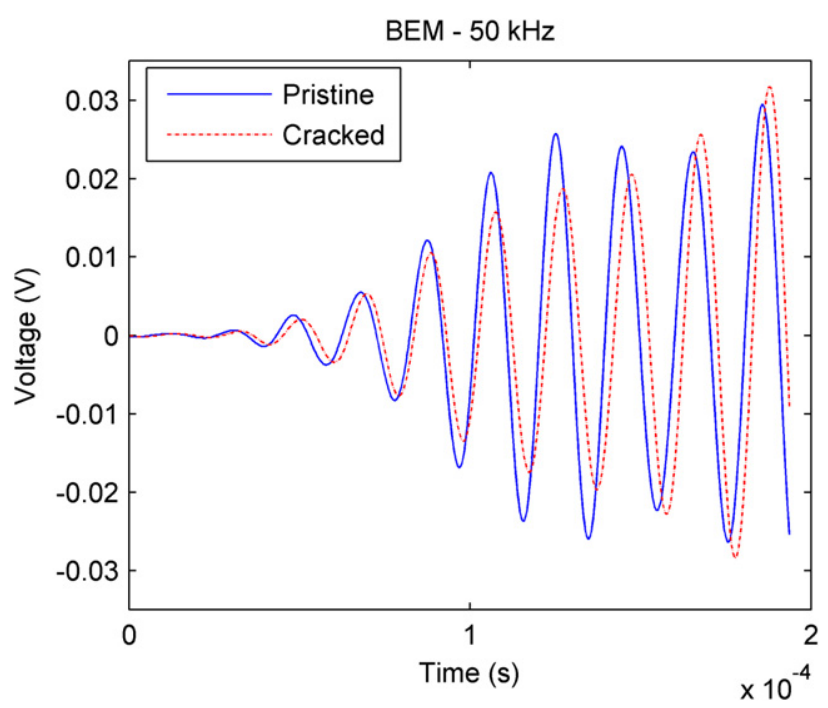

Figure 12. Comparison of signals from BEM simulations for $50 \mathrm{kHz}$ actuation frequency.

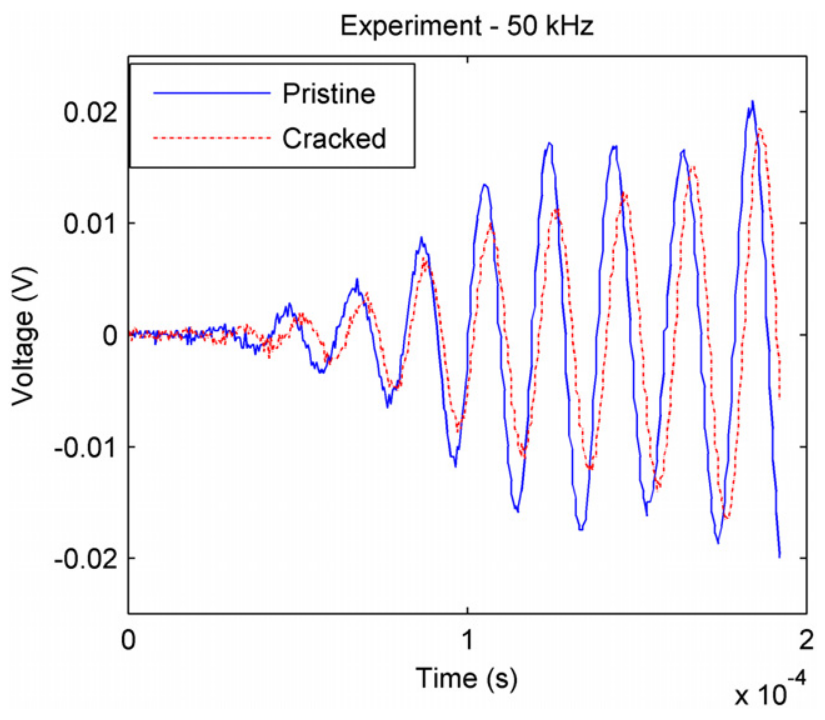

Figure 13. Comparison of signals from experiment for $50 \mathrm{kHz}$ actuation frequency.

by the manufacturers who provided the data. This would result in differences in velocities of the transmitted waves and voltage readings. Finally, other factors such as climate, energy dissipation and errors embedded in the experimental setup may equally contribute to the discrepancy between the experimental and the BEM results.

Nevertheless, what is important in SHM applications is the difference between the signals acquired from the pristine and the damaged structures. In figures 12-15, attenuations in signals can be seen when cracks are present. Also, the fact that attenuation is greater for signals with higher frequency complies with the understanding that signals with higher frequency are more sensitive to the presence of damage sites [26]. 


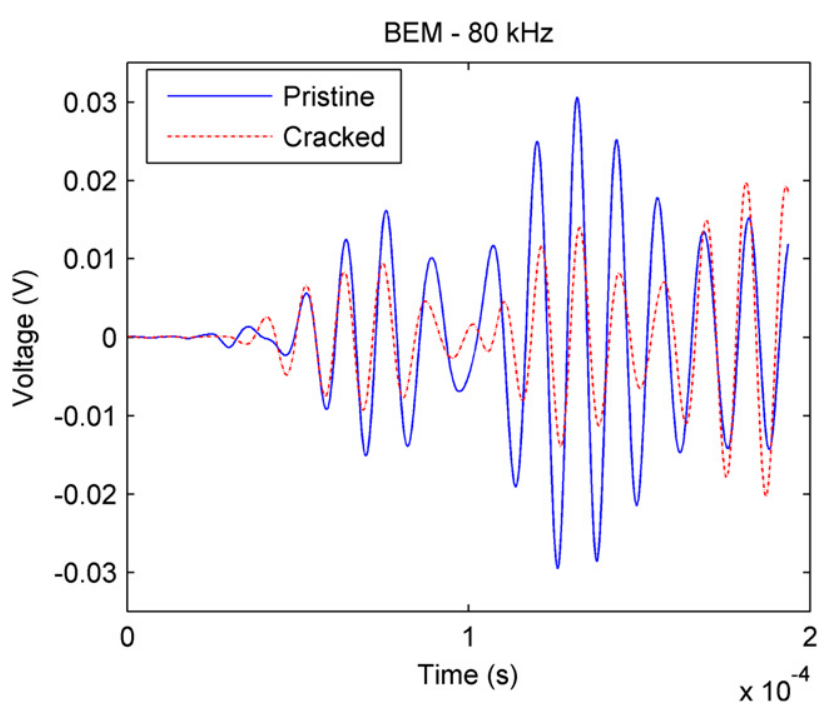

Figure 14. Comparison of signals from BEM simulations for $80 \mathrm{kHz}$ actuation frequency.

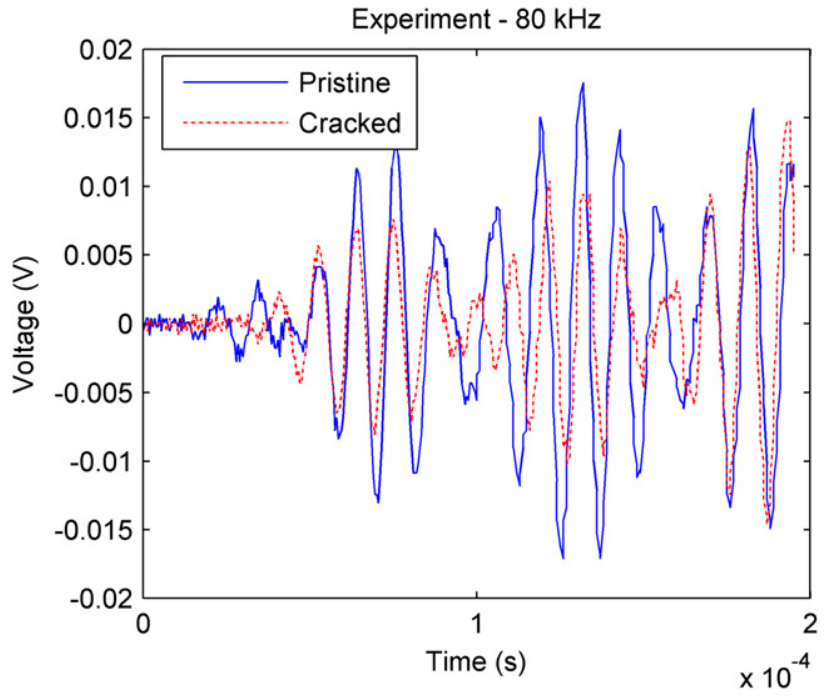

Figure 15. Comparison of signals from experiment for $80 \mathrm{kHz}$ actuation frequency.

\subsection{Parametric study}

The experimentally validated DBEM formulation is employed to investigate the effects of a couple of parameters in SHM applications. The actuation signals used have a central frequency of $80 \mathrm{kHz}$ and a peak voltage of $10 \mathrm{~V}$.

5.5.1. Crack depth The signals obtained from beams with cracks of two different depths- 6.25 and $18.75 \mathrm{~mm}$-are compared with that obtained from a pristine beam. For all three cases, both the actuator and the sensor are placed $3 \mathrm{~cm}$ away from the crack.

Two general trends can be observed from the signals plotted in figure 16. As the crack deepens, the signal becomes more attenuated and the time of arrival becomes longer. The first phenomenon is due to the fact the crack

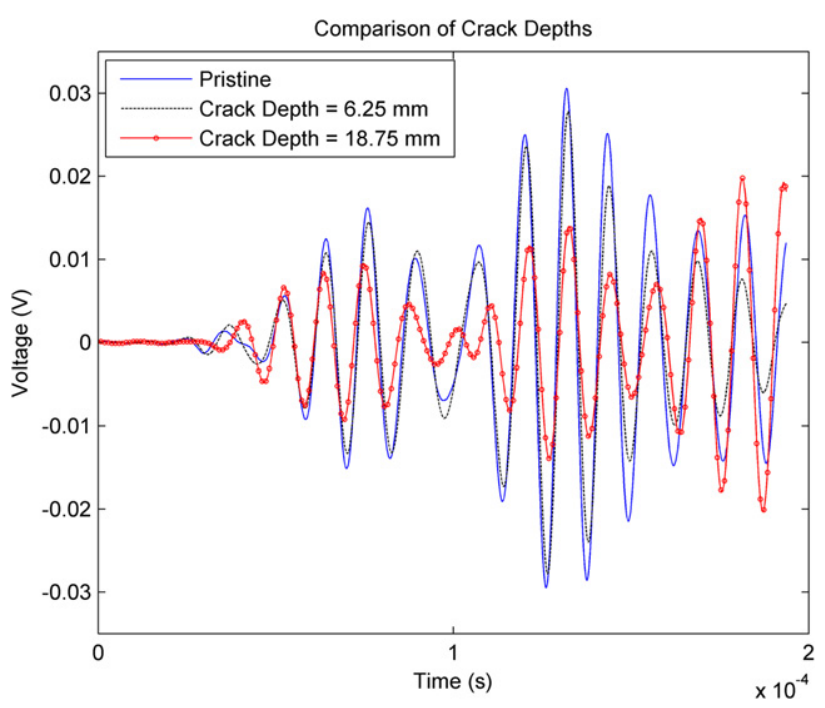

Figure 16. Comparison of signals from BEM simulations for different crack depths. (The actuation signal has a central frequency of $80 \mathrm{kHz}$ and a peak voltage of $10 \mathrm{~V}$.)

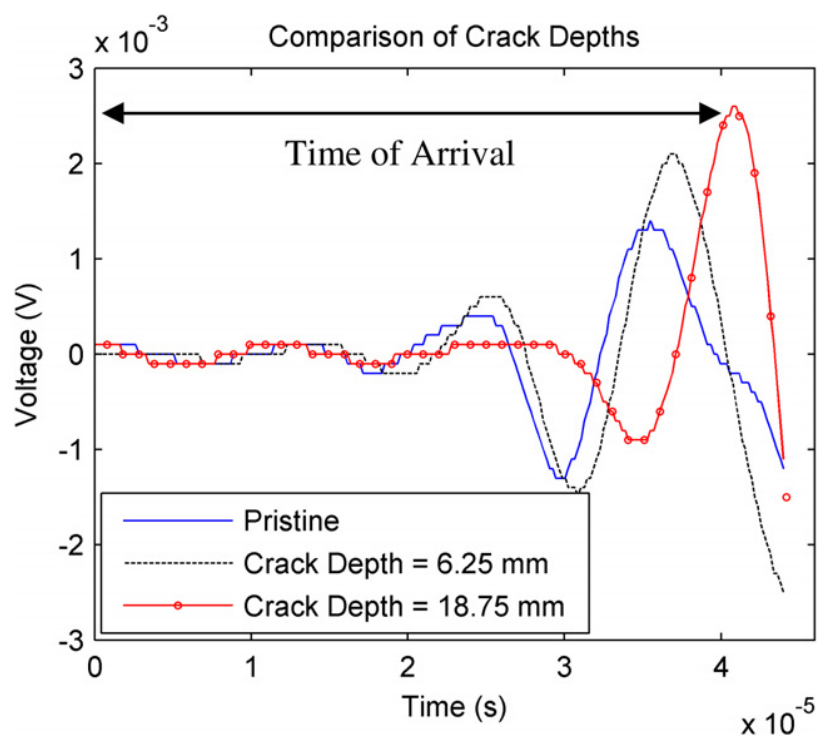

Figure 17. Comparison of time of arrival from BEM simulations for different crack depths $(80 \mathrm{kHz}, 10 \mathrm{~V})$.

acts as an obstacle which dissipates some of the energy carried by the transmitted waves and thus attenuates the amplitude. The second phenomenon, as shown in figure 17, is because of the extra distance of separation between the piezoelectric transducers, introduced by the discontinuity of the material. Both phenomena have been reported in previous research $[27,28]$.

5.5.2. Arrangement of transducer Three different transducer arrangements, in which both the actuator and the sensor are placed 3, 5 and $7 \mathrm{~cm}$ away from the crack, are examined for their ability to monitor the presence of cracks. All cracks used in this study have a depth of $18.75 \mathrm{~mm}$.

First of all, by looking at the signals from the pristine beams, it can be seen that as the distance of separation 


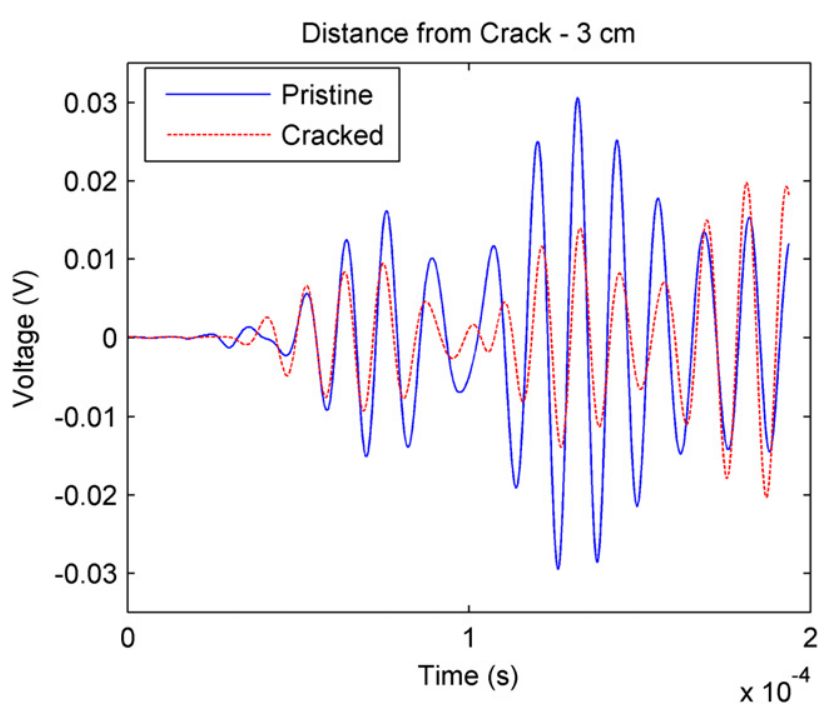

Figure 18. Comparison of signals from BEM simulation for a distance of $3 \mathrm{~cm}$ from the crack $(80 \mathrm{kHz}, 10 \mathrm{~V})$.

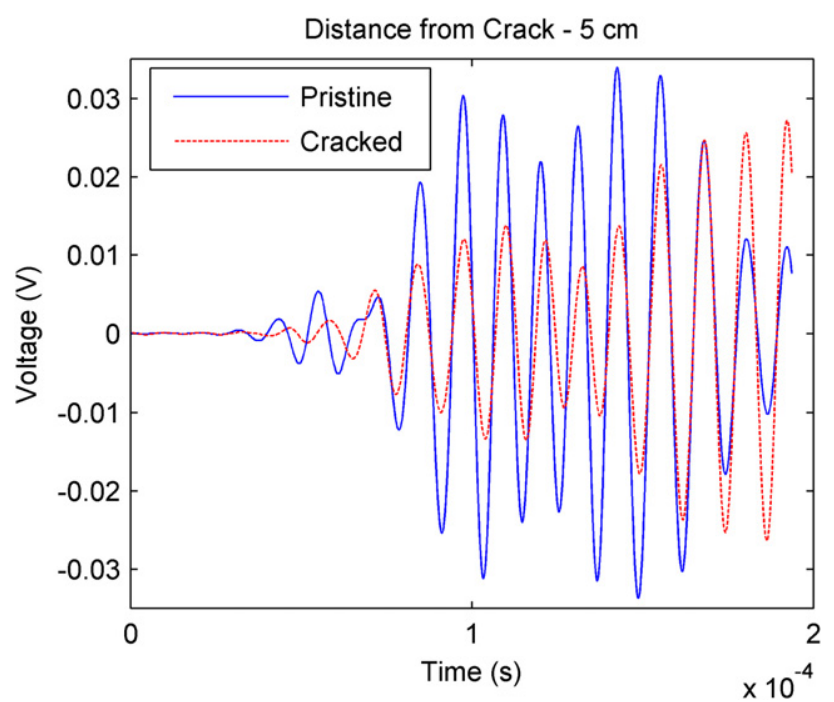

Figure 19. Comparison of signals from BEM simulation for a distance of $5 \mathrm{~cm}$ from the crack $(80 \mathrm{kHz}, 10 \mathrm{~V})$.

between the piezoelectric transducers increases, the time of arrival becomes longer, as expected. Also, the directly transmitted waves and the reflections become less isolated since the transducers are closer to the boundaries.

In all three figures, the differences between the signals obtained from the pristine and the damaged beams are significant. The presence of damage leads to later time of arrival, as explained in the previous section. Also, as the piezoelectric transducers become more separated, phase delay, in addition to attenuation in amplitude, can be observed.

\subsection{Wave propagation}

In figure 21, contour plots of vertical displacements and their corresponding instants in sensor signals are shown for both the pristine and the cracked beams. The actuation signal used has a central frequency of $80 \mathrm{kHz}$ and a peak voltage of $10 \mathrm{~V}$.

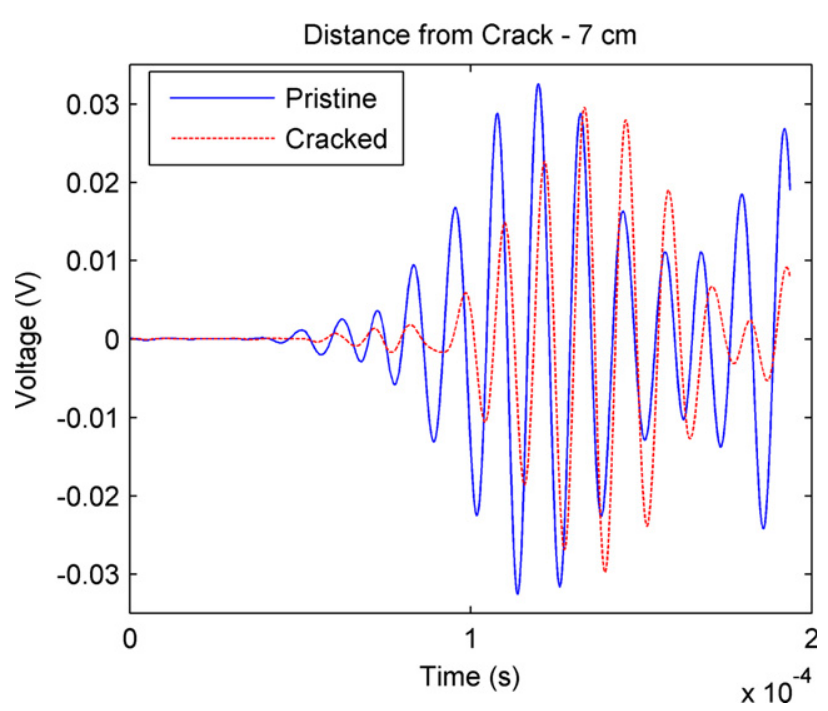

Figure 20. Comparison of signals from BEM simulation for a distance of $7 \mathrm{~cm}$ from the crack $(80 \mathrm{kHz}, 10 \mathrm{~V})$.

For the pristine beam, the three frames show, respectively, the beginning of the actuation, the intermediate propagation and when the waves are reflected from the boundaries. It can be deduced that the second batch of waves in the sensor signal and thereafter are the combinations of reflections from all boundaries.

For the cracked beam, the frames are dedicated to the time period in which the actuated waves encounter the crack. From the three consecutive instants, it can be seen that the waves are not able to pass through the crack directly but have to negotiate around it. This gives explanation to how the energy carried by the waves is dissipated when confronted by a crack. Also, after the waves have travelled around the crack, they immediately begin to occupy the whole body again, as reported by Jian et al [27].

\section{Conclusions}

In this paper, the first dynamic BEM for modelling smart structures, instrumented with piezoelectric transducers for SHM applications, is introduced. The generalized 3D dynamic model for piezoelectricity takes into account the full electro-mechanical relationship and the effect of inertia. The dedicated actuator and sensor models are then developed by applying the appropriate boundary conditions, and with some algebraic manipulation, they are purposely expressed in terms of BEM variables to allow for direct coupling with the host structure. The elastodynamic analysis of the whole structure is achieved with boundary integrals in Laplace domain, and the response in time domain is obtained with inverse Laplace transform.

The BEM is validated against FEM and experimental results. The specimens include a pristine and a cracked beam. The agreement between the sensor signals obtained from BEM and FEM simulations and from experiments is outstanding. The experimentally validated formulation is used to perform a series of parametric study in order to demonstrate 

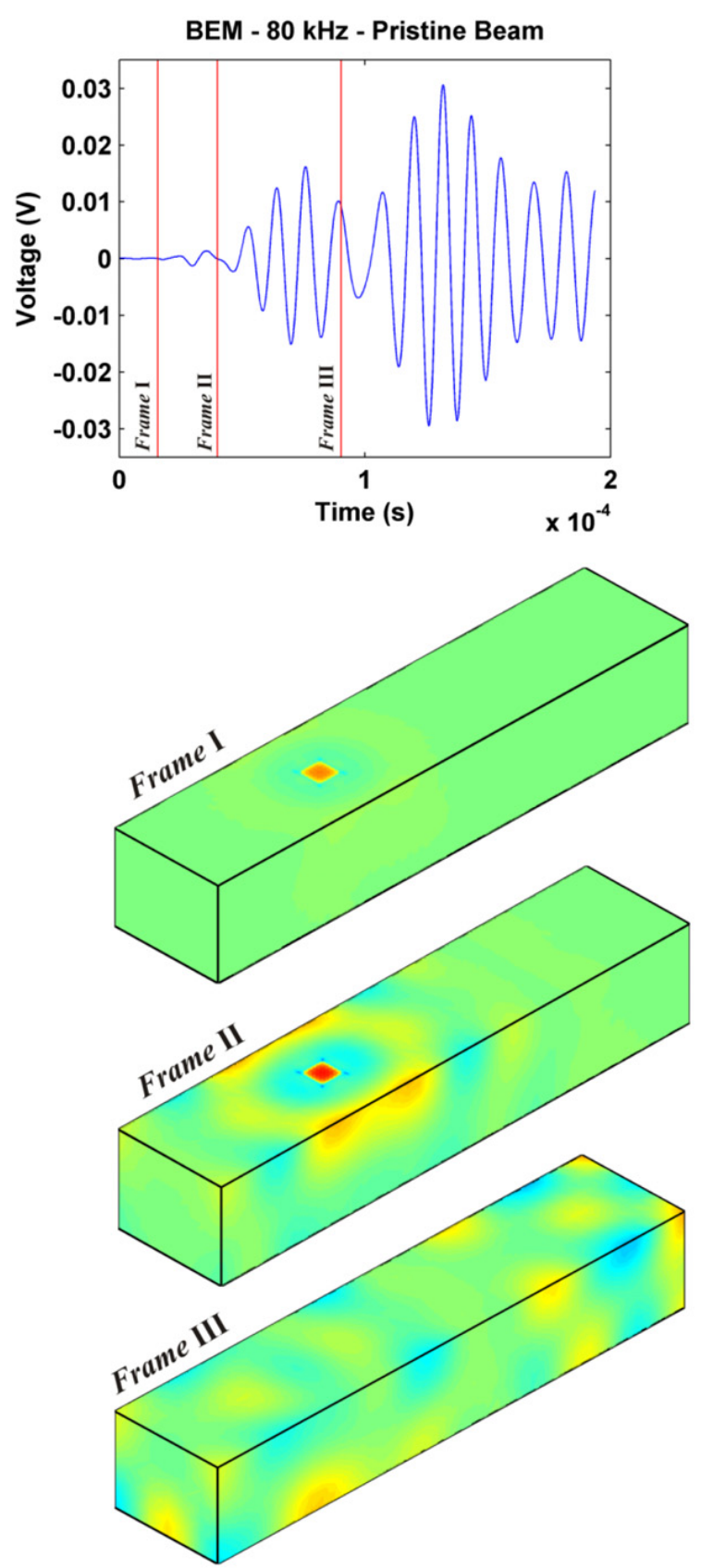
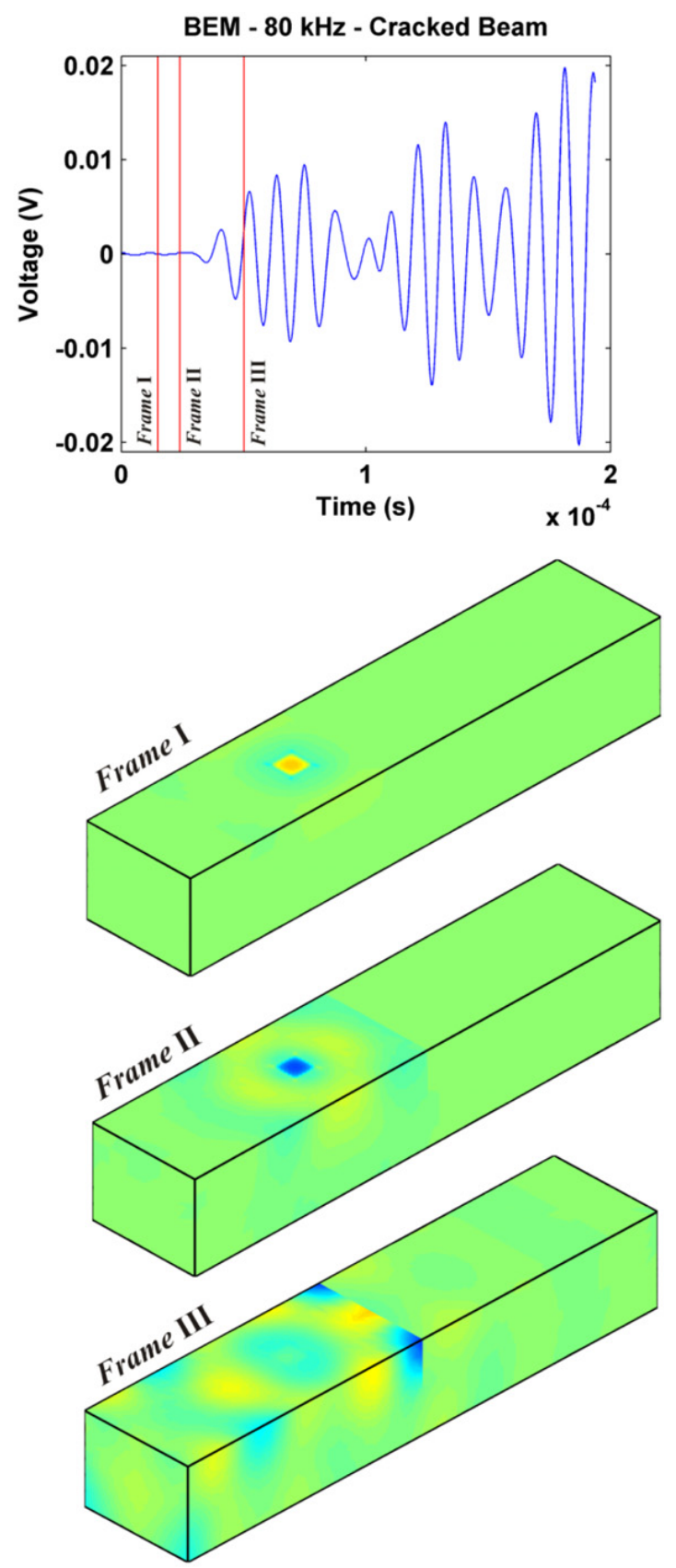

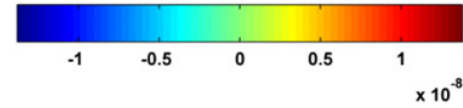

Figure 21. Contour plots of vertical displacements and indications of frames for pristine and cracked beams. (The actuation signal has a central frequency of $80 \mathrm{kHz}$ and a peak voltage of $10 \mathrm{~V}$.)

its feasibility in SHM developments. The capability of the model for studying wave propagation and scattering of waves by cracks is also shown.

As mentioned before, one reason for the difference between the amplitudes of the signals obtained from BEM and FEM simulations and experiments is the presence of damping in the response of the structure. Therefore, a future work that could be carried out is the inclusion of damping effects in the numerical model. Jin et al [29] provided an overview of the damping models that have been incorporated with one-, two- and three-dimensional BEM in time and frequency domains. More recently, Mazzotti et al [30], in their 2.5D BEM, introduced material damping using the bulk attenuation coefficient. Nevertheless, the predetermination of the overall damping of a structure is not straightforward.

In the experimental results, ambient white noise can be seen in the sensor signals. Although the modelling of white noise has been done with BEM in acoustics [31], it, in 
solid mechanics, is still under investigation. In the future, it would be ideal to incorporate white noise in the numerical model because structures in service would experience an even higher level of noise than specimens in laboratory. With the fundamental solutions presented in this paper, the high frequency components of white noise would lead to an enormous amount of computation and numerical instability. Therefore, other BEM formulations, such as time-domain BEM and dual reciprocity BEM, should be investigated for their abilities in handling high frequencies. Also, it would be of interest to implement wide-band excitation signals, such as white noise and swept sine, which have been used in piezoelectric based SHM application [32], with BEM.

Compared to FEM, BEM is numerically more stable and computationally less expensive. In particular, DBEM possesses the natural advantage in modelling material discontinuity, which is essential in SHM developments. With the rapid advances in SHM in recent years, the need for an accurate and efficient model for understanding the behaviour of smart structures is becoming increasingly important. This paper is the first step towards finding such an alternative to FEM.

\section{Appendix A}

The fundamental solutions in equation (4) are given by

$$
\begin{aligned}
U_{i j}\left(\boldsymbol{x}^{\prime}, \boldsymbol{x}, s\right)= & \frac{1}{4 \pi G}\left(\psi \delta_{i j}-\chi r_{, i} r_{, j}\right) \\
T_{i j}\left(\boldsymbol{x}^{\prime}, \boldsymbol{x}, s\right)= & \frac{1}{4 \pi}\left[\left(\psi_{, r}-\frac{\chi}{r}\right)\left(\frac{\delta r}{\delta n} \delta_{i j}+r_{, j} n_{i}\right)-2 \frac{\chi}{r}\right. \\
& \times\left(n_{j} r_{, i}-2 r_{, i} r_{, j} \frac{\delta r}{\delta n}\right)-2 \chi_{, r} r_{, i} r_{, j} \frac{\delta r}{\delta n} \\
& \left.+\left(\frac{c_{1}^{2}}{c_{2}^{2}}-2\right)\left(\psi_{, r}-\chi_{, r}-2 \frac{\chi}{r}\right) n_{j} r_{, i}\right]
\end{aligned}
$$

where

$$
\begin{aligned}
\psi & =\frac{\mathrm{e}^{-\frac{s r}{c_{2}}}}{r}+\frac{1+\frac{p s}{c_{2}}}{\left(\frac{p s}{c_{2}}\right)^{2}} \frac{\mathrm{e}^{-\frac{s r}{c_{2}}}}{r}-\frac{c_{2}^{2}}{c_{1}^{2}} \frac{1+\frac{p s}{c_{1}}}{\left(\frac{p s}{c_{1}}\right)^{2}} \frac{\mathrm{e}^{-\frac{s r}{c_{1}}}}{r} \\
\chi & =3 \psi-2 \frac{\mathrm{e}^{-\frac{s r}{c_{2}}}}{r}-\frac{c_{2}^{2}}{c_{1}^{2}} \frac{\mathrm{e}^{-\frac{s r}{c_{1}}}}{r} \\
r & =\left|\boldsymbol{x}^{\prime}-\boldsymbol{x}\right| \\
G & =\frac{E}{2(1-v)} .
\end{aligned}
$$

The kernel functions in equation (5) are given by

$$
\begin{aligned}
& U_{k i j}\left(\boldsymbol{x}^{\prime}, \boldsymbol{x}, s\right) \\
& =\frac{1}{4 \pi}\left[2\left(\chi, r-2 \frac{\chi}{r}\right) r_{, i} r_{, j} r_{, k}+2 \frac{\chi}{r} \delta_{i j} r_{, k}-\left(\psi_{, r}-\frac{\chi}{r}\right)\right. \\
& \left.\quad \times\left(\delta_{i k} r_{, j}-\delta_{j k} r_{, i}\right)-\frac{\lambda}{G}\left(\psi_{, r}-\chi, r-2 \frac{\chi}{r}\right) \delta_{i j} r_{, k}\right]
\end{aligned}
$$

$$
\begin{aligned}
& T_{k i j}\left(\boldsymbol{x}^{\prime}, \boldsymbol{x}, s\right) \\
& =\frac{G}{4 \pi}\left\{\frac { \delta r } { \delta n } \left[4\left(\chi, r r-5 \frac{\chi, r}{r}+8 \frac{\chi}{r^{2}}\right) r_{, i} r_{, j} r_{, k}\right.\right. \\
& -\left(\psi_{, r r}-\frac{\psi_{, r}}{r}-3 \frac{\chi, r}{r}+6 \frac{\chi}{r^{2}}\right)\left(\delta_{i k} r_{, j}+\delta_{j k} r_{, i}\right) \\
& +2\left(2 \frac{\chi, r}{r}-4 \frac{\chi}{r^{2}}+\frac{\lambda}{G}\left(\chi, r r+\frac{\chi, r}{r}-4 \frac{\chi}{r^{2}}\right.\right. \\
& \left.\left.\left.-\psi_{, r r}+\frac{\psi_{, r}}{r}\right)\right) \delta_{i j} r_{, k}\right]+2\left(2 \frac{\chi, r}{r}-4 \frac{\chi}{r^{2}}\right. \\
& \left.+\frac{\lambda}{G}\left(\chi_{, r r}+\frac{\chi_{, r}}{r}-4 \frac{\chi}{r^{2}}-\psi_{, r r}+\frac{\psi_{, r}}{r}\right)\right) r_{, i} r_{, j} n_{k} \\
& -\left(\psi_{, r r}-\frac{\psi_{, r}}{r}-3 \frac{\chi, r}{r}+6 \frac{\chi}{r^{2}}\right)\left(r_{, j} n_{i}+r_{, i} n_{j}\right) r_{, k} \\
& +\left(4 \frac{\chi}{r^{2}}+\frac{\lambda}{G}\left(4 \frac{\chi, r}{r}+8 \frac{\chi}{r^{2}}-4 \frac{\psi, r}{r}\right)+\frac{\lambda^{2}}{G^{2}}\right. \\
& \left.\times\left(\chi_{, r r}+4 \frac{\chi_{, r}}{r}+2 \frac{\chi}{r^{2}}-\psi_{, r r}-2 \frac{\psi_{, r}}{r}\right)\right) \delta_{i j} n_{k} \\
& \left.-2\left(\frac{\psi, r}{r}-\frac{\chi}{r^{2}}\right)\left(\delta_{k j} n_{i}+\delta_{k i} n_{j}\right)\right\}
\end{aligned}
$$

where

$$
\lambda=\frac{E v}{(1+v)(1-2 v)} .
$$

\section{Appendix B}

The linear differential operators in equation (12) are given by

$$
\boldsymbol{D}_{\alpha}=\left[\begin{array}{cccc}
\frac{\partial}{\partial_{1}} & 0 & 0 & 0 \\
0 & \frac{\partial}{\partial_{2}} & 0 & 0 \\
\frac{\partial}{\partial_{2}} & \frac{\partial}{\partial} & 0 & 0 \\
0 & 0 & 0 & \frac{\partial}{\partial_{1}} \\
0 & 0 & 0 & \frac{\partial}{\partial_{2}}
\end{array}\right] \quad \boldsymbol{D}_{\beta}=\left[\begin{array}{cccc}
0 & 0 & \frac{\partial}{\partial_{1}} & 0 \\
0 & 0 & \frac{\partial}{\partial_{2}} & 0 \\
0 & 0 & 0 & 0 \\
0 & 0 & 0 & 0
\end{array}\right] .
$$

The energy density in equation (15) is given by

$$
\begin{aligned}
\boldsymbol{\omega}\left(\boldsymbol{U}, \boldsymbol{\Sigma}_{z}\right)= & \boldsymbol{\Sigma}_{z}^{\mathrm{T}}\left(\boldsymbol{D}_{\beta}+\boldsymbol{I} \frac{\partial}{\partial x_{3}}\right) \boldsymbol{U} \\
& +\frac{1}{2}\left(\left(\boldsymbol{D}_{\alpha} \boldsymbol{U}\right)^{\mathrm{T}} \boldsymbol{\Phi}_{p p} \boldsymbol{D}_{\alpha} \boldsymbol{U}-\boldsymbol{U}^{\mathrm{T}} \boldsymbol{\Omega} \boldsymbol{U}\right) \\
& -\frac{1}{2} \boldsymbol{\Sigma}_{z}^{\mathrm{T}} \boldsymbol{\Phi}_{z z} \boldsymbol{\Sigma}_{z}+\boldsymbol{\Sigma}_{z}^{\mathrm{T}} \boldsymbol{\Phi}_{z p} \boldsymbol{D}_{\alpha} \boldsymbol{U}
\end{aligned}
$$

where

$$
\boldsymbol{\Phi}_{z z}=\boldsymbol{R}_{z z}^{-1}=\left[\begin{array}{cccc}
k_{1} & 0 & 0 & 0 \\
0 & k_{2} & 0 & 0 \\
0 & 0 & k_{3} & k_{4} \\
0 & 0 & k_{4} & k_{5}
\end{array}\right]
$$




$$
\begin{array}{rl}
\boldsymbol{\Phi}_{z p}=\boldsymbol{R}_{z z}^{-1} \boldsymbol{R}_{z p}=\left[\begin{array}{ccccc}
0 & 0 & 0 & k_{6} & 0 \\
0 & 0 & 0 & 0 & k_{7} \\
k_{8} & k_{9} & 0 & 0 & 0 \\
k_{10} & k_{11} & 0 & 0 & 0
\end{array}\right] & \begin{array}{c}
\text { Appendix C } \\
\text { In the field of BEM, the most often used approach for inverse } \\
\text { Laplace transform is the Durbin's method [33], whose formula } \\
\text { is written as }
\end{array} \\
\boldsymbol{\Phi}_{p p}=\boldsymbol{R}_{z z}-\boldsymbol{R}_{p z} \boldsymbol{R}_{z z}^{-1} \boldsymbol{R}_{z p} & f(t)=2 \frac{\mathrm{e}^{\mathrm{a} t}}{T}\left\{-\frac{1}{2} \operatorname{Re}(F(a))+\sum_{k=0}^{L}\left[\operatorname{Re}\left(F\left(a+\mathrm{i} \frac{2 k \pi}{T}\right)\right)\right.\right. \\
=\left[\begin{array}{ccccc}
k_{12} & k_{13} & 0 & 0 & 0 \\
k_{13} & k_{14} & 0 & 0 & 0 \\
0 & 0 & k_{15} & 0 & 0 \\
0 & 0 & 0 & k_{16} & 0 \\
0 & 0 & 0 & 0 & k_{17}
\end{array}\right] & \times \cos \left(\frac{2 k \pi t}{T}\right)-\operatorname{Im}\left(F\left(a+\mathrm{i} \frac{2 k \pi}{T}\right)\right)
\end{array}
$$$$
\boldsymbol{\Omega}=\left[\begin{array}{cccc}
\rho \omega^{2} & 0 & 0 & 0 \\
0 & \rho \omega^{2} & 0 & 0 \\
0 & 0 & \rho \omega^{2} & 0 \\
0 & 0 & 0 & 0
\end{array}\right] \text {. }
$$

where $L$ is the total number of Laplace parameters and $T$ is the time period of interest. The term $a+\mathrm{i} \frac{2 k \pi}{T}$ is the discretized form of the Laplace parameter $a+\mathrm{i} \omega$, indicating that the frequency range of interest is divided into a number of increments. Therefore, the number of Laplace parameters must be sufficient for fully covering the frequency range of the response. Also, according to Zhao [34], the real part of the Laplace parameter $a$, which affects the error of the inversion, is determined by

$$
a=\max \left(\operatorname{Re}\left(p_{i}\right)\right)+\frac{5}{T}
$$

where $p_{i}$ are the singularities of the signal to be inverted.

\section{References}

[1] Giurgiutiu V 2005 Tuned Lamb wave excitation and detection with piezoelectric wafer active sensors for structural health monitoring J. Intell. Mater. Syst. Struct. 16 291-305

[2] Ihn J-B and Chang F-K 2004 Detection and monitoring of hidden fatigue crack growth using a built-in piezoelectric sensor/actuator network: I. Diagnostics Smart Mater. Struct. 13609

[3] Lee J-R, Takatsubo J and Toyama N 2007 Disbond monitoring at wing stringer tip based on built-in ultrasonic transducers and a pulsed laser Smart Mater. Struct. 161025

[4] Mallardo V, Aliabadi M H and Sharif Khodaei Z 2013 Optimal sensor positioning for impact localization in smart composite panels J. Intell. Mater. Syst. Struct. 24 559-73

[5] Sharif Khodaei Z, Ghajari M and Aliabadi M H 2012 Determination of impact location on composite stiffened panels Smart Mater. Struct. 21105026

[6] Zhao X et al 2007 Active health monitoring of an aircraft wing with embedded piezoelectric sensor/actuator network: I. Defect detection, localization and growth monitoring Smart Mater. Struct. 161208

[7] Ghajari M, Sharif Khodaei Z, Aliabadi M H and Apicella A 2013 Identification of impact force for smart composite stiffened panels Smart Mater. Struct. 22085014

[8] Chee C Y, Tong L and Steven G P 1998 A review on the modelling of piezoelectric sensors and actuators incorporated in intelligent structures J. Intell. Mater. Syst. Struct. 9 3-19

where

$$
\begin{aligned}
& \boldsymbol{N}_{x}=\frac{\partial \boldsymbol{N}_{\mathrm{s}}}{\partial x} \\
& \boldsymbol{N}_{y}=\frac{\partial \boldsymbol{N}_{\mathrm{s}}}{\partial y} .
\end{aligned}
$$

[9] Crawley E F and De Luis J 2012 Use of piezoelectric actuators as elements of intelligent structures AIAA J. $\mathbf{2 5}$

[10] Crawley E F and Lazarus K B 2012 Induced strain actuation of isotropic and anisotropic plates AIAA J. 29

[11] Raghavan A and Cesnik C E 2005 Finite-dimensional piezoelectric transducer modeling for guided wave based structural health monitoring Smart Mater. Struct. 141448 
[12] Lin X and Yuan F 2001 Diagnostic Lamb waves in an integrated piezoelectric sensor/actuator plate: analytical and experimental studies Smart Mater. Struct. 10907

[13] Aliabadi M H 2002 The Boundary Element Method. Applications in Solids and Structures vol 2 (Chichester: Wiley)

[14] Portela A, Aliabadi M H and Rooke D 1992 The dual boundary element method: effective implementation for crack problems Int. J. Numer. Methods Eng. 33 1269-87

[15] Mi Y and Aliabadi M H 1992 Dual boundary element method for three-dimensional fracture mechanics analysis Eng. Anal. Bound. Elem. 10 161-71

[16] Wen P H, Aliabadi M H and Young A 1999 Dual boundary element methods for three-dimensional dynamic crack problems J. Strain Anal. Eng. Des. 34 373-94

[17] Davì G and Milazzo A 2001 Multidomain boundary integral formulation for piezoelectric materials fracture mechanics Int. J. Solids Struct. 38 7065-78

[18] Wünsche M, Zhang C, García-Sánchez F, Sáez A, Sladek J and Sladek V 2011 Dynamic crack analysis in piezoelectric solids with non-linear electrical and mechanical boundary conditions by a time-domain BEM Comput. Methods Appl. Mech. Eng. 200 2848-58

[19] Leme S, Aliabadi M H, Bezerra L and Partridge P 2007 An investigation into active strain transfer analysis in a piezoceramic sensor system for structural health monitoring using the dual boundary element method Struct. Health Monit. 3 121-32

[20] Benedetti I, Aliabadi M H and Milazzo A 2010 A fast BEM for the analysis of damaged structures with bonded piezoelectric sensors Comput. Methods Appl. Mech. Eng. 199 490-501

[21] Alaimo A, Milazzo A and Orlando C 2013 Numerical analysis of a piezoelectric structural health monitoring system for composite flange-skin delamination detection Compos. Struct. $100343-55$

[22] Wen P H, Aliabadi M H and Rooke D 1998 Cracks in three dimensions: a dynamic dual boundary element analysis Comput. Methods Appl. Mech. Eng. 167 139-51

[23] Guang-hui Q, Jia-jun Q and Yan-hong L 2005 Modified HR mixed variational principle for magnetoelectroelastic bodies and state-vector equation Appl. Math. Mech. 26 722-8
[24] Benedetti I and Aliabadi M H 2010 A fast hierarchical dual boundary element method for three-dimensional elastodynamic crack problems Int. J. Numer. Methods Eng. 84 1038-67

[25] Benedetti I, Aliabadi M H and Davi G 2008 A fast 3D dual boundary element method based on hierarchical matrices Int. J. Solids Struct. 45 2355-76

[26] Doebling S W, Farrar C R, Prime M B and Shevitz D W 1996 Damage Identification and Health Monitoring of Structural and Mechanical Systems from Changes in their Vibration Characteristics: A Literature Review (NM: Los Alamos National Lab.)

[27] Jian X, Dixon S, Guo N and Edwards R 2007 Rayleigh wave interaction with surface-breaking cracks J. Appl. Phys. 101064906

[28] Pecorari C 2001 Scattering of a Rayleigh wave by a surface-breaking crack with faces in partial contact Wave Motion 33 259-70

[29] Jin F, Pekau O and Zhang C H 2001 A 2D time-domain boundary element method with damping Int. J. Numer. Methods Eng. 51 647-61

[30] Mazzotti M, Bartoli I, Marzani A and Viola E 2013 A coupled SAFE-2.5 D BEM approach for the dispersion analysis of damped leaky guided waves in embedded waveguides of arbitrary cross-section Ultrasonics

[31] Brancati A, Aliabadi M H and Benedetti I 2009 Hierarchical adaptive cross approximation GMRES technique for solution of acoustic problems using the boundary element method Comput. Model. Eng. Sci. 43149

[32] Biemans C, Staszewski W, Boller C and Tomlinson G 2001 Crack detection in metallic structures using broadband excitation of acousto-ultrasonics J. Intell. Mater. Syst. Struct. 12 589-97

[33] Durbin F 1974 Numerical inversion of Laplace transforms: an efficient improvement to Dubner and Abate's method Comput. J. 17 371-6

[34] Zhao X 2004 An efficient approach for the numerical inversion of Laplace transform and its application in dynamic fracture analysis of a piezoelectric laminate Int. $J$. Solids Struct. 41 3653-74 
Queries for IOP paper 480774

Journal: $\quad$ SMS

Author: $\quad$ F Zou et al

Short title: A boundary element model for structural

health monitoring using piezoelectric transducers

Page 1

Query 1:

Author: Please check that the author names and affiliations as given are spelt correctly.

\section{Page 1}

\section{Query 2:}

Author: Please be aware that the colour figures in this article will only appear in colour in the Web version. If you require colour in the printed journal and have not previously arranged it, please contact the Production Editor now.

\section{Page 2}

Query 3:

Author: Bold and non-bold characters used inconsistently throughout the text. Please check.

\section{Page 4}

Query 4:

Author: Superscript 'italic bold T' given in equation (30) and in similar cases changed to 'roman non bold T'. Please check.

\section{Page 14}

\section{Query 5:}

Author: Please check the details for any journal references that do not have a blue link as they may contain some incorrect information. Pale purple links are used for references to arXiv e-prints.

\section{Page 14}

Query 6:

Author: [9, 10]: Please provide the page/article number.

\section{Page 15}

Query 7:

Author: [19]: Please check the journal title given here.

\section{Page 15}

Query 8.

Author: [26]: Please provide city of publisher. And also check the publisher name given here.

\section{Page 15}

Query 9:

Author: [30]: Please provide volume and page/article number.

\section{Uncited Figures}

Figures $5,8,9,10,11,13,14,18,19$ and 20 are not cited in text.

\section{Uncited Tables}

Tables 1 and 2 are not cited in text. 